\title{
Long-distance entanglement of spin qubits via quantum Hall edge states
}

\author{
Guang Yang, ${ }^{1}$ Chen-Hsuan Hsu, ${ }^{1}$ Peter Stano, ${ }^{1}$ Jelena Klinovaja, ${ }^{2}$ and Daniel Loss ${ }^{1,2}$ \\ ${ }^{1}$ RIKEN Center for Emergent Matter Science, Wako, Saitama 351-0198, Japan \\ ${ }^{2}$ Department of Physics, University of Basel, Klingelbergstrasse 82, CH-4056 Basel, Switzerland \\ (Received 6 October 2015; revised manuscript received 12 January 2016; published 1 February 2016)
}

\begin{abstract}
The implementation of a functional quantum computer involves entangling and coherent manipulation of a large number of qubits. For qubits based on electron spins confined in quantum dots, which are among the most investigated solid-state qubits at present, architectural challenges are often encountered in the design of quantum circuits attempting to assemble the qubits within the very limited space available. Here, we provide a solution to such challenges based on an approach to realizing entanglement of spin qubits over long distances. We show that long-range Ruderman-Kittel-Kasuya-Yosida interaction of confined electron spins can be established by quantum Hall edge states, leading to an exchange coupling of spin qubits. The coupling is anisotropic and can be either Ising type or XY type, depending on the spin polarization of the edge state. Such a property, combined with the dependence of the electron spin susceptibility on the chirality of the edge state, can be utilized to gain valuable insights into the topological nature of various quantum Hall states.
\end{abstract}

DOI: 10.1103/PhysRevB.93.075301

\section{INTRODUCTION}

Quantum computers, exploiting entanglement and superposition of quantum mechanical states, promise much better performance than classical computers tackling a collection of important mathematical problems [1]. Over the past few decades, a variety of solid-state systems have been studied for the implementation of qubits, the building blocks of a quantum computer. Among such systems, a very promising candidate [2] makes use of the spin of electrons confined in semiconductor quantum dots (QDs). In that scheme, entanglement of qubits is achieved through the direct exchange interaction between confined electrons, and manipulation of individual qubits can be realized by magnetic or electrical means [3]. Recent advances in QD technology have established long coherence times [4] exceeding $0.2 \mathrm{~ms}$ and fast gate-operation times [3] on the order of tens of nanoseconds for spin qubits in QDs.

With the great progress in the development of quality spin qubits, scalability becomes the next major challenge towards building a functional quantum computer capable of performing fault-tolerant quantum computing [5]. The implementation of quantum-error-correction algorithms [6] requires that the system reach a size of several thousands of qubits. In practice, however, one faces tremendous difficulties in assembling so many spin qubits, among which entanglement must be selectively established and maintained. Indeed, the nearest-neighbor nature of the direct exchange interaction, the primary source of entanglement, restricts drastically access of each qubit to the rest of the system and thus the space that can be used for installing the quantum circuits. A natural way to overcome such difficulties is to employ means of entangling spin qubits over long distances, which creates extra space for wiring the quantum circuits. In principle, this may be achieved by coupling the spin qubits to an electromagnetic cavity [7-10], a floating metallic gate [11], or a dipolar ferromagnet [12]. Recently, it was shown that coupling of distant spin qubits can also be realized via photon-assisted cotunneling [13].

In this paper, we propose a mechanism to achieve longdistance entanglement of spin qubits. We make use of the
Ruderman-Kittel-Kasuya-Yosida (RKKY) interaction [14-16] between confined electron spins in QDs, mediated by the conducting edge states of quantum Hall $(\mathrm{QH})$ liquids [17], to which the QDs are tunnel coupled [18]. The spin qubit coupling obtained in such a way is particularly interesting. Depending on whether the edge state is spin polarized or not, the induced coupling between the spin qubits can be Ising type and perpendicular to the plane of the system, or XY type and in plane. This offers great versatility in the design of large-scale quantum circuits. The advantage of using $\mathrm{QH}$ edge states is twofold. First, the edge states and the QDs can be formed in the same material (by top gates) such as a two-dimensional electron gas (2DEG) in GaAs heterostructures. Second, the $\mathrm{QH}$ edge states are topologically stable and thus much more robust against disorder effects compared to one-dimensional (1D) conduction channels in quantum wires. Moreover, we find that the spin susceptibility of QH edge states manifests the inequivalence between the opposite directions, "clockwise" and "counterclockwise", along the QH edge. In chiral edge states, conduction electrons propagate in only one direction, leading to a "rectified" spin susceptibility in the propagation direction of electrons. In nonchiral edge states, the spin susceptibility is nonzero in both directions along the $\mathrm{QH}$ edge, but with different magnitudes. The spin susceptibility has the same type of anisotropy as the coupling between qubits. Thus, measuring the spatial dependence of the spin susceptibility [19] can serve as a powerful probe of the chirality and spin polarization of the edge state, and thus of the topological order [17] in a QH liquid.

\section{MODEL}

We now discuss the physics of RKKY interaction mediated by $\mathrm{QH}$ edge states. The basic setup is shown in Fig. 1. Two QDs are placed adjacent to a $\mathrm{QH}$ liquid, separated by a distance $L$ and labeled by the site index $i=1,2$. Conduction electrons in the QH edge state can tunnel into and out of the QDs [18] and thus can interact with the localized spins in them. This establishes coupling between the QH edge and the QDs. 


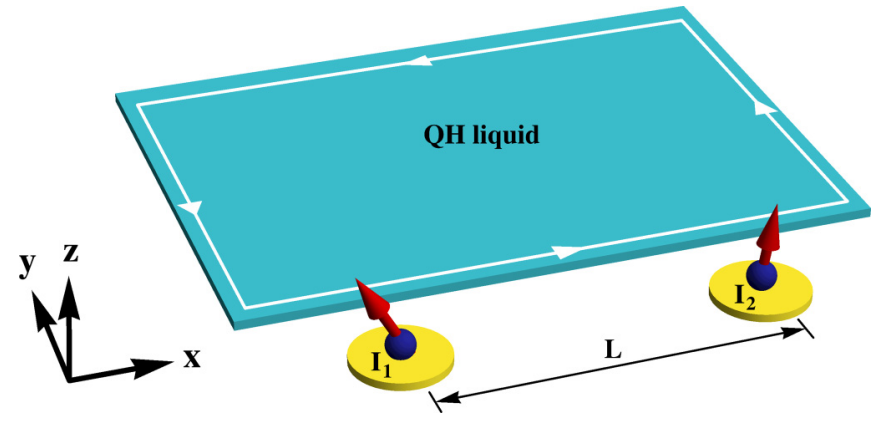

FIG. 1. The basic setup consisting of two QDs (yellow disks) tunnel coupled to the edge (white lines and arrows) of a $\mathrm{QH}$ liquid (blue sheet) confined in the $x-y$ plane. In general, the $\mathrm{QH}$ edge may support multiple edge modes, propagating in the same or opposite direction(s), which we do not depict explicitly. The QDs are separated by a distance $L$ along the QH edge. Each QD contains a single electron (blue spheres), whose spin (red arrows) serves as a qubit. The coupling strength between the QDs and the QH edge is controlled by gates (not shown). We assume no direct interaction between the localized electron spins $\mathbf{I}_{1}$ and $\mathbf{I}_{2}$ in the QDs.

For simplicity, we treat the QDs as two spatial points. The Hamiltonian describing such a system has the form

$$
H=H_{\text {edge }}+\sum_{i=1,2} \Gamma_{i} \mathbf{S}_{i} \cdot \mathbf{I}_{i},
$$

where $H_{\text {edge }}$ is the Hamiltonian of conduction electrons in the edge state, $\mathbf{I}_{i}=\left(I_{i}^{x}, I_{i}^{y}, I_{i}^{z}\right)$ denotes the localized spin in the $i$ th $\mathrm{QD}$, and $\mathbf{S}_{i}=\left(S_{i}^{x}, S_{i}^{y}, S_{i}^{z}\right)$ denotes the spin of conduction electrons coupled to $\mathbf{I}_{i}$, with coupling strength $\Gamma_{i}$. Experimentally, $\Gamma_{i}$ can be tuned by gating. We define $\mathbf{S}_{i}$ to be the spin density in the edge state multiplied by the confinement length of the QDs. For the setup, we assume $L$ is large so that there is no direct interaction between the spins in the QDs.

In the weak tunnel coupling regime such that $\Gamma_{i} \ll E_{F}$, where $E_{F}$ is the Fermi energy of conduction electrons, the dynamics of the spins in the QDs effectively decouples from that of the conduction electrons. In such a case, one can derive an effective Hamiltonian for the spins in QDs, valid in the adiabatic regime, by performing a Schrieffer-Wolff transformation $[20,21]$ of Eq. (1) followed by tracing out the degrees of freedom of conduction electrons (see Appendix A for the derivation of effective Hamiltonian and a discussion of adiabaticity),

$$
H_{\mathrm{eff}}=\sum_{i j, \alpha \beta} J_{i j}^{\alpha \beta} I_{i}^{\alpha} I_{j}^{\beta}-\sum_{i} \mathbf{B}_{i} \cdot \mathbf{I}_{i},
$$

where the spin-component indices $\alpha, \beta=x, y, z$. The first term is the RKKY interaction, with $J_{i j}^{\alpha \beta}=\Gamma_{i} \Gamma_{j} \chi_{i j}^{\alpha \beta} / 2$. Here, $\chi_{i j}^{\alpha \beta}$ is the static spin susceptibility of conduction electrons, $\chi_{i j}^{\alpha \beta}=-i \int_{0}^{\infty} d t e^{-\eta t}\left\langle\left[S_{i}^{\alpha}(t), S_{j}^{\beta}(0)\right]\right\rangle$, where $\eta=0^{+}$and $\langle\ldots\rangle$ denotes the average determined by $H_{\text {edge }}$. Physically, conduction electrons in the vicinity of a QD develop a spin-density oscillation due to their interaction with the spin in the QD. This spin-density response, determined by $\chi_{i j}^{\alpha \beta}$, can be perceived by the spins in other QDs coupled to the QH edge. In this way, the RKKY interaction is established. For spin-unpolarized
QH states, we assume $\left\langle S_{i}^{x}\right\rangle=\left\langle S_{i}^{y}\right\rangle=\left\langle S_{i}^{z}\right\rangle=0$, such that $\chi_{i j}^{\alpha \beta}=\delta^{\alpha \beta} \chi_{i j}^{\alpha \alpha}$. On the other hand, the in-plane spin operators $S_{i}^{x}, S_{i}^{y}$ are less relevant (in the renormalization group sense) than the out-of-plane ones $S_{i}^{z}$ in a $\mathrm{QH}$ state with full spin polarization, as we discuss in the following. In this case, we set $S_{i}^{x}=S_{i}^{y}=0$ and hence $\chi_{i j}^{\alpha \beta}=\delta^{\alpha z} \delta^{\beta z} \chi_{i j}^{z z}$. Thus, in general we have $J_{i j}^{\alpha \beta}=\delta^{\alpha \beta} J_{i j}^{\alpha \alpha}$. The RKKY interaction leads to an effective exchange coupling $\mathcal{J}^{\alpha}=J_{12}^{\alpha \alpha}+J_{21}^{\alpha \alpha}$, as a function of the interdot distance $L$, between the localized spins $I_{1}^{\alpha}$ and $I_{2}^{\alpha}$. The effective onsite Zeeman fields $\mathbf{B}_{i}$ are a direct consequence of time-reversal (TR) symmetry breaking in $\mathrm{QH}$ systems, $B_{i}^{\alpha}=\left(\Gamma_{i}^{2} / 4\right) \int_{0}^{\infty} d t e^{-\eta t} \epsilon^{\alpha \beta \gamma}\left\langle\left\{S_{i}^{\beta}(t), S_{i}^{\gamma}(0)\right\}\right\rangle$. We find that $B_{i}^{\alpha}=\delta^{\alpha z} B_{i}^{z}$ in spin-unpolarized QH states and $\mathbf{B}_{i}=0$ in spin-polarized $\mathrm{QH}$ states (for more details and estimates, we refer to Appendix A).

\section{RKKY INTERACTION IN VARIOUS QH STATES}

The RKKY interaction in Eq. (2) is by nature long ranged and can be used as an approach to entangle spin qubits over long distances. Thus, it is important to understand how the interaction looks in various $\mathrm{QH}$ systems. To this end, it is convenient to adopt a continuum description of the $\mathrm{QH}$ edge states that is well approximated by the chiral Luttinger liquid (LL) model at low energy [17]. In general, the edge of a QH liquid may support (electron-) density-fluctuation modes as well as Majorana fermions (zero modes), with the action

$$
\begin{aligned}
S_{\text {edge }}= & \int d x d t\left[\sum_{I J} \frac{1}{4 \pi}\left(K_{I J} \partial_{t} \phi_{I} \partial_{x} \phi_{J}-V_{I J} \partial_{x} \phi_{I} \partial_{x} \phi_{J}\right)\right. \\
& \left.+\sum_{K} i \lambda_{K}\left(\partial_{t}-v_{K} \partial_{x}\right) \lambda_{K}\right]
\end{aligned}
$$

written in the bosonization language [17] (throughout the paper we set $\hbar=1)$. The bosonic fields $\phi_{I}$ describe the density modes, and $\lambda_{K}$ denote the Majorana fermions. The symmetric matrix $K_{I J}$ encodes the topological properties of the QH state, while the positive-definite symmetric matrix $V_{I J}$ specifies the velocities and interactions of $\phi_{I}$. The parameter $v_{K}$ is the velocity of $\lambda_{K}: v_{K}>0\left(v_{K}<0\right)$ if the $\lambda_{K}$ is left moving (right moving).

Upon passing to the continuum limit, we replace the spin operators $S_{i}^{\alpha}(t) / l$ with spin-density operators $S^{\alpha}\left(x_{i}, t\right)$, where $l$ is the confinement length of the QDs and $x_{i}$ is the position of the $i$ th QD. The nonvanishing components of the spin susceptibility are given by $\chi_{i j}^{\alpha \alpha}=-i l^{2} \int_{0}^{\infty} d t e^{-\eta t}\left\langle\left[S^{\alpha}\left(x_{i}, t\right), S^{\alpha}\left(x_{j}, 0\right)\right]\right\rangle$. Assuming translation invariance along the $\mathrm{QH}$ edge, which is justified for clean samples, we may further write $\chi_{i j}^{\alpha \alpha}=$ $\chi^{\alpha \alpha}\left(x_{i}-x_{j}\right)$ [22], where

$$
\chi^{\alpha \alpha}(x)=2 l^{2} \int_{0}^{\infty} d t e^{-\eta t} \operatorname{Im}\left\langle\mathcal{T} S^{\alpha}(x, t) S^{\alpha}(0,0)\right\rangle,
$$

with $\mathcal{T}$ the time-ordering operator. The correlators are evaluated in the zero-temperature limit. We define

$$
S^{\alpha}(x, t)=\frac{1}{2} \sum_{\sigma \sigma^{\prime}} \psi_{\sigma}^{\dagger}(x, t) \sigma_{\sigma \sigma^{\prime}}^{\alpha} \psi_{\sigma^{\prime}}(x, t),
$$


where $\psi_{\sigma}=\sum_{\mu} \psi_{\sigma}^{\mu}$ is the sum of the most relevant electron operators $\psi_{\sigma}^{\mu}$ with spin $\sigma=\uparrow, \downarrow$ on the QH edge.

The number of $\psi_{\uparrow}^{\mu}$ operators is not necessarily equal to that of $\psi_{\downarrow}^{\mu}$ operators since TR symmetry is broken. For instance, the most relevant electron operators have the same spin in a spin-polarized QH state, so that $S^{x}=S^{y}=0$. This is in contrast to the situation in 1D systems where TR symmetry is present [23,24]. Using bosonization, we express $S^{\alpha}$ in terms of the fields $\phi_{I}$ and $\lambda_{K}$, and compute the spin susceptibility.

We sketch the calculation of the spin susceptibility for a generic $\mathrm{QH}$ edge state (for particular examples, see Appendix B). First of all, we assume separation of charged and neutral degrees of freedom in the $\mathrm{QH}$ edge state. This phenomenon, as has been demonstrated experimentally in a number of QH systems [25,26], results from strong Coulomb interaction among the elementary density modes $\phi_{I}$ and resembles "charge-spin separation" in a generic TR-invariant $1 \mathrm{D}$ system [22]. As a result, the physical modes that propagate on the $\mathrm{QH}$ edge are the charged and neutral collective modes as well as Majorana fermions. The physical parameters relevant to experiment are the velocities and interactions of these propagating modes, whose magnitudes are set by different energy scales in the QH system. For instance, the charged-mode velocity, determined by the dominant Coulomb energy scale, is much greater than the velocity of neutral mode and other parameters [25]. We make use of this fact in our calculation. For a moment, we consider the case of two density modes in the edge theory [see Eq. (3)]. To compute the correlators in Eq. (4), we define a new set of fields which diagonalize the action of the density modes $\phi_{I}$. The action takes the form

$$
\begin{aligned}
S_{\text {density }}= & \int d x d t \frac{1}{4 \pi}\left[\partial_{t} \phi_{+} \partial_{x} \phi_{+}+\varepsilon \partial_{t} \phi_{-} \partial_{x} \phi_{-}\right. \\
& \left.-v_{+} \partial_{x} \phi_{+} \partial_{x} \phi_{+}-v_{-} \partial_{x} \phi_{-} \partial_{x} \phi_{-}\right]
\end{aligned}
$$

in the basis of new fields $\phi_{+}$and $\phi_{-}$. Here, $\varepsilon=1(\varepsilon=-1)$ if the edge states are chiral (nonchiral) and $v_{+}, v_{-}>0$. New velocities $v_{+}$and $v_{-}$are well approximated by the velocities of the physical charged mode and neutral mode, respectively, so that $v_{+} \gg v_{-}$. Upon expressing the spin-density operators in terms of the free fields $\phi_{+}, \phi_{-}$, and $\lambda_{K}$, it is straightforward to compute the correlators

$$
\begin{aligned}
\left\langle\mathcal{T} S^{\alpha}(x, t) S^{\alpha}(0,0)\right\rangle \propto & \cos (\Delta k x)\left[\frac{1}{\delta+i\left(t+x / v_{+}\right)}\right]^{g_{+}^{\alpha}} \\
\times & {\left[\frac{1}{\delta+i\left(t+\varepsilon x / v_{-}\right)}\right]^{g_{-}^{\alpha}}, }
\end{aligned}
$$

where $\delta>0$ is an infinitesimal and $\Delta k$ is the gauge-invariant momentum difference between the edge modes. The case $\Delta k=0$ corresponds to the scattering of an edge mode with itself. Here, we have omitted the terms that are less relevant, and assumed $\left|v_{K}\right|=v_{-}$as both of the velocities are determined by less dominant energy scales in the system. The exponents $g_{+}^{\alpha}, g_{-}^{\alpha}$ are functions of the matrices $K_{I J}$ and $V_{I J}$ and as we show $0<g_{+}^{\alpha} \ll 1$ and $g_{-}^{\alpha}>1$ (see Appendix B for the expressions in different $\mathrm{QH}$ states). Evaluating the time integral in Eq. (4), we obtain $\chi^{\alpha \alpha}(x)$, which in general may contain multiple terms for different momentum differences. We keep only the most relevant terms.
The various $\mathrm{QH}$ states can be divided into three types: (i) those with a chiral edge state containing a single density mode, such as the Laughlin states at filling factors $v=1 / \mathrm{m}$, where $m$ is an odd integer; (ii) those with a chiral edge state containing multiple interacting density modes, such as the $\mathrm{QH}$ state at $v=2$; (iii) those with a nonchiral edge state, such as the particle-hole dual states [27] of Laughlin states.

For QH states of type (i), we find $\chi^{\alpha \alpha}(x)=0$, taking into account the most relevant spin operators in the edge state. Thus, to the lowest order, the RKKY interaction cannot be established. Physically, the vanishing spin susceptibility reflects the homogeneous electronic structure in an independent $\mathrm{QH}$ edge mode, a property originating from the incompressibility of the QH liquid which prevents the formation of electronic spin texture. In reality, however, a small nonzero spin susceptibility may still be measured, due to higher-order processes involving virtual transitions to edge states in higher Landau levels.

In $\mathrm{QH}$ edge states of types (ii) and (iii), the spin susceptibility is nonzero to the lowest order. In these cases, the interedge interactions introduce inhomogeneous degrees of freedom ("noise") to the stream of conduction electrons, allowing for the development of spin-density oscillations. We find

$$
\chi^{\alpha \alpha}(x)=\frac{\cos (\Delta k x)}{|x|^{g^{\alpha}}} \Theta(-x) C^{\alpha}\left(g^{\alpha}, \boldsymbol{v}\right)
$$

for left-moving type (ii) edge states, where $g^{\alpha}=g_{+}^{\alpha}+g_{-}^{\alpha}-1$, $\Theta(x)$ is the Heaviside step function, and $C^{\alpha}\left(\boldsymbol{g}^{\alpha}, \boldsymbol{v}\right)$ are functions of $\boldsymbol{g}^{\alpha}=\left(g_{+}^{\alpha}, g_{-}^{\alpha}\right)$ and $\boldsymbol{v}=\left(v_{+}, v_{-}\right)$, whose explicit definitions are given in Appendix B. If the edge state is right moving, one replaces $\Theta(-x)$ with $\Theta(x)$, and sends $\boldsymbol{v} \rightarrow-\boldsymbol{v}$ in $C^{\alpha}\left(g^{\alpha}, \boldsymbol{v}\right)$. These findings suggest that the spin susceptibility in type (ii) edge states is "rectified", i.e., directed in the downstream direction of the propagation of conduction electrons [see Fig. 2(a)], where left- and right-moving directions are defined with respect to the lower edge of the $\mathrm{QH}$ liquid [the same in Fig. 2(b)]. This result is not surprising and can be understood also intuitively. In a left-moving edge state, conduction electrons move in the $-x$ direction, leading to the factor $\Theta(-x)$ in the expression of $\chi^{\alpha \alpha}(x)$. Formally, such an interesting form of the spin susceptibility is a manifestation of

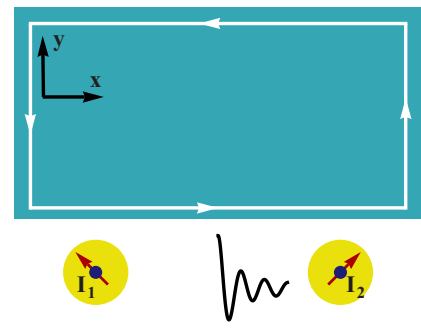

(a)

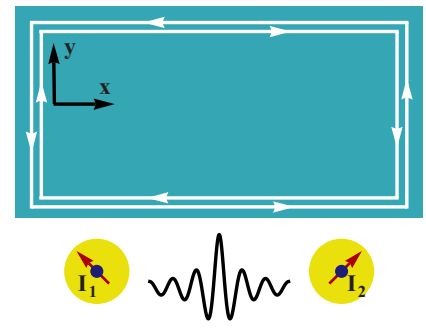

(b)
FIG. 2. Spin susceptibility in QH edge states of (a) type (ii) and (b) type (iii). For the type (ii) case, the spin susceptibility is directed in the propagation direction of the edge modes. For the type (iii) case, the spin susceptibility is nonzero in both directions along the $\mathrm{QH}$ edge. 
the causality principle in 1D chiral systems, where information is transported one way and novel physical rules can emerge, e.g., see Ref. [28] for fluctuation-dissipation relations in chiral QH systems.

Lastly, we find

$$
\chi^{\alpha \alpha}(x)=\frac{\cos (\Delta k x)}{|x|^{g^{\alpha}}}\left\{\Theta(x) C_{>}^{\alpha}\left(g^{\alpha}, \boldsymbol{v}\right)+\Theta(-x) C_{<}^{\alpha}\left(\boldsymbol{g}^{\alpha}, \boldsymbol{v}\right)\right\}
$$

for type (iii) edge states, where $C_{>}^{\alpha}\left(\boldsymbol{g}^{\alpha}, \boldsymbol{v}\right)$ and $C_{<}^{\alpha}\left(\boldsymbol{g}^{\alpha}, \boldsymbol{v}\right)$ are functions of $\boldsymbol{g}^{\alpha}$ and $\boldsymbol{v}$, defined in Appendix B. The spin susceptibility in this case is "both-way", as shown in Fig. 2(b), with different magnitudes in the $+x$ and $-x$ directions, i.e., $C_{>}^{\alpha}\left(\boldsymbol{g}^{\alpha}, \boldsymbol{v}\right) \neq C_{<}^{\alpha}\left(\boldsymbol{g}^{\alpha}, \boldsymbol{v}\right)$. This again reflects the inequivalence between left moving and right-moving edge modes. Imagining now the chirality of all edge modes is reverted, e.g., by TR operation, the profile of the spin susceptibility should also be reverted. Indeed, we find that $C_{>}^{\alpha}\left(\boldsymbol{g}^{\alpha}, \boldsymbol{v}\right)$ are related to $C_{<}^{\alpha}\left(\boldsymbol{g}^{\alpha}, \boldsymbol{v}\right)$ by the exchange of arguments $v_{+} \leftrightarrow v_{-}$and $g_{+}^{\alpha} \leftrightarrow g_{-}^{\alpha}$, which technically carries out the chirality-reverting procedure (see Appendix B). In the above discussion, we have assumed that spin excitations do not extend into the $L_{0}-L$ part of the $\mathrm{QH}$ edge, where $L_{0}$ is the total edge length. In practice, this is realized by grounding the $L_{0}-L$ part or by choosing the sample such that $L_{0} \gg L$.

The exponents $g^{\alpha}$, where $\alpha=x, y, z$, determine how the RKKY interaction scales with distance. In Table I, we list them in different QH states. In general, $g^{\alpha}$ depend on both the chirality and spin polarization of the $\mathrm{QH}$ edge state. For chiral edge states, i.e., those of type (ii), these exponents are integral invariants depending on the topological order of the bulk liquid, whereas for nonchiral edge states they are nonuniversal and depend on the parameters in the Hamiltonian. In the latter case, we write $g^{\alpha}=g_{0}^{\alpha}+\delta g^{\alpha}$, where $g_{0}^{\alpha}$ is the integer part of $g^{\alpha}$. As shown in Appendix B, $\delta g^{\alpha} / g_{0}^{\alpha} \ll 1$ for all the nonchiral edge states in the table, assuming "charge-neutral separation" on the edge. Moreover, we find that the in-plane components of the RKKY interaction vanish in a spin-polarized $\mathrm{QH}$ state, leading to an Ising-type exchange coupling of spin qubits. On the other hand, the RKKY interaction has zero out-of-plane component and equal in-plane components in a spin-unpolarized QH state, which is XY type. This suggests that a transformation of the anisotropy type of the RKKY interaction may be observed in the $\mathrm{QH}$ liquid at $v=\frac{2}{3}$, which was found to be spin unpolarized at low fields and spin polarized at high fields [29].

The QH state at $v=\frac{5}{2}$ is also of special interest. We consider both Abelian and non-Abelian topological orders proposed to describe this state. The former include the Halperin 331 state [30] and 113 state [31], and the latter include the Moore-Read (Pfaffian) state [32], the anti-Pfaffian state $[33,34]$, and the $\mathrm{SU}(2)_{2}$ state [35]. The 331 and 113 states can be both spin polarized and unpolarized, just like the $v=\frac{2}{3}$ $\mathrm{QH}$ state. The Pfaffian state, like the Laughlin states, supports a single density mode on the edge and thus has vanishing RKKY interaction. The particle-hole dual state of the Pfaffian state, the anti-Pfaffian state, has a nonchiral edge state and a noninteger scaling exponent. For the $\mathrm{SU}(2)_{2}$ state, we assume that the Majorana fermion and the neutral collective mode propagate at different velocities, as they should in reality, which is necessary to obtain a nonvanishing scaling exponent. Such careful treatment is not essential for other $v=\frac{5}{2}$ states. We have assumed that the RKKY interaction is mediated solely by the fractional edge modes in the second Landau level, while the integer edge modes in the lowest Landau level do not play a role. Experimentally, this can be fulfilled, using the fact that edge modes in different Landau levels are spatially separated [36]. For instance, the QDs in Fig. 1 can be moved out of the plane of the $\mathrm{QH}$ liquid and formed in a second two- or quasi-one-dimensional electron gas in the vertical direction [37-40], such that they are in tunnel contact with the fractional edge modes but far away from the integer edge modes. The coupling between the integer edge and the QDs and the interaction between the integer edge and the fractional edge can be neglected to a good approximation.

\section{DISCUSSION}

Let us estimate the coupling between the two spin qubits in Fig. 1, given by $\mathcal{J}^{\alpha}=\Gamma_{1} \Gamma_{2}\left\{\chi^{\alpha \alpha}(L)+\chi^{\alpha \alpha}(-L)\right\} / 2$. In Appendix B, we obtain the dimensional part $\left[\chi^{\alpha \alpha}(x)\right]$ of the spin susceptibility

$$
\left[\chi^{\alpha \alpha}(x)\right] \simeq l^{2} a^{g^{\alpha}-1}|x|^{-g^{\alpha}} / v_{-}
$$

for both type (ii) and type (iii) edge states, where $a$ is the lattice constant of the underlying material hosting the $\mathrm{QH}$ system. For example, let us consider the $\mathrm{QH}$ state at $v=2$, realized in GaAs heterostructures. We have $a=0.565 \mathrm{~nm}$ for GaAs, $g^{x}=g^{y}=1$, and $v_{-} \simeq 10^{4} \mathrm{~m} / \mathrm{s}$ [25]. Using $\Gamma_{1}=$ $\Gamma_{2}=\Gamma \simeq 0.1 \mathrm{meV}$ and $l=30 \mathrm{~nm}$ (see Appendix $\mathrm{C}$ for the estimates), we find $\mathcal{J}^{x}=\mathcal{J}^{y} \simeq 1 \mu \mathrm{eV}$ for $L=1 \mu \mathrm{m}$. This is about one order of magnitude smaller than the direct exchange strength $\mathcal{J}_{\text {direct }} \simeq 10-100 \mu \mathrm{eV}$ in typical GaAs double QDs [2] and is experimentally measurable. The RKKY interaction established by $\mathrm{QH}$ edge states thus provides a way to realize entangled quantum gates over mesoscopic distances. The implementation of two-qubit gates using Hamiltonians of the form of Eq. (2) is well known: see, e.g., Ref. [2] (footnote 13) for Ising-type coupling and Ref. [7] for XY-type coupling. The $\mu \mathrm{eV}$ exchange strength converts to gate-operation times of the order of nanoseconds, which is well below the coherence times [3] of spin qubits.

It is interesting to compare the RKKY interaction in $\mathrm{QH}$ edge states with that in semiconductor quantum wires. Assuming spin-rotation symmetry, the dimensional part $\left[\chi_{\mathrm{w}}(x)\right]$ of the spin susceptibility in quantum wires can be found in Ref. [23]. The ratio

$$
r^{\alpha}(x)=\frac{\left[\chi^{\alpha \alpha}(x)\right]}{\left[\chi_{\mathrm{w}}(x)\right]}=\frac{v_{F}}{v_{-}}\left(\frac{a}{|x|}\right)^{g^{\alpha}-g_{\mathrm{w}}}
$$

characterizes the relative strength of the RKKY interaction in the two sorts of systems, where $v_{F}$ is the Fermi velocity in the quantum wire and $g_{\mathrm{w}}$ depends on the interaction of electrons. In noninteracting case, $g_{\mathrm{w}}=1$. Consider the $v=2 \mathrm{QH}$ edge state and GaAs quantum wire. We find $r^{x}(L)=r^{y}(L) \simeq 1.5$ for $L=1 \mu \mathrm{m}$, using $g_{\mathrm{w}}=0.75$ and $v_{F} \simeq 10^{5} \mathrm{~m} / \mathrm{s}$ [23]. In principle, quantum wires can also be used to mediated RKKY interaction between spin qubits. However, using QH edge states offers more advantages. From technical aspect, the edge 
TABLE I. Scaling exponents $g^{\alpha}$ and anisotropy type of the RKKY interaction in various QH states. An overline is used to indicate a spin-polarized state, e.g., $\frac{2}{3}$ denotes the spin-polarized QH state at $v=\frac{2}{3}$. We consider several topological orders at $v=\frac{5}{2}$, including both Abelian ones (the 331 state and the 113 state, denoted as $331 / \overline{331}$ and $113 / \overline{113}$, respectively) and non-Abelian ones (the Pfaffian state, the anti-Pfaffian state, and the $\mathrm{SU}(2)_{2}$ state, denoted as $\overline{\mathrm{Pf}}, \overline{\mathrm{APf}}$, and $\overline{\mathrm{SU}(2)_{2}}$, respectively). The 331 state and the 113 state both have spin-unpolarized and spin-polarized versions. For chiral edge states, the exponents are integers and we add arrows to indicate that the spin susceptibility is nonzero only in the downstream direction. For nonchiral edge states, the exponents are nonintegers and we enter the integer parts $g_{0}^{\alpha}$ of the exponents. We put "-_" in the entry if the corresponding component of the spin susceptibility (and thus that of the RKKY interaction) vanishes. The RKKY interaction is XY type in spin-unpolarized states and Ising type in spin-polarized states.

\begin{tabular}{lccccccccccc}
\hline \hline QH state & $\overline{1 / m}$ & 2 & $\frac{2}{3}$ & $\overline{\frac{2}{3}}$ & 331 & $\overline{331}$ & 113 & $\overline{113}$ & $\overline{\mathrm{Pf}}$ & $\overline{\mathrm{APf}}$ & $\overline{\mathrm{SU}(2)_{2}}$ \\
\hline$g^{x}$ & - & $\overrightarrow{1}$ & 1 & - & $\overrightarrow{3}$ & - & 3 & - & - & - \\
$g^{y}$ & - & $\overrightarrow{1}$ & 1 & - & $\overrightarrow{3}$ & - & 3 & - & - & - \\
$g^{z}$ & - & - & - & 1 & - & $\overrightarrow{3}$ & - & 3 & - & 1 & - \\
RKKY type & & $\mathrm{XY}$ & $\mathrm{XY}$ & Ising & $\mathrm{XY}$ & Ising & $\mathrm{XY}$ & Ising & Ising & Ising \\
\hline \hline
\end{tabular}

states and the spin qubits can be realized in the same material, for instance, in a $2 \mathrm{DEG}$ in GaAs heterostructures, which is more experimentally accessible than a setup with quantum wires. More importantly, the topologically protected QH edge states are more immune to disorder effects and perturbations in the system than quantum wires. This guarantees a better quality of the long-distance quantum gates.

Our discussions so far have focused on the RKKY interaction between spin qubits. Interestingly, the treatments can also be applied to obtain the RKKY interaction between nuclear spins embedded in the $1 \mathrm{D} \mathrm{QH}$ edge state (see also Ref. [41]). To this end, let $\Gamma_{i} \rightarrow A / N$ and $\mathbf{I}_{i} \rightarrow \tilde{\mathbf{I}}_{i}$ in Eq. (1) and the following equations, where $A$ is the hyperfine coupling constant, $N$ is the number of nuclear spins in a cross section (labeled by $i$ ) of the $\mathrm{QH}$ edge, and $\tilde{\mathbf{I}}_{i}$ is the total nuclear spin operator in a given cross section. Given a nonchiral edge state with both spin-up and -down electrons, e.g., the spin-unpolarized state at $v=\frac{2}{3}$, the nuclear spins may form a helical magnetic order [23] at low temperatures, induced by the RKKY interaction. The nuclear magnetic order acts back on the electronic system by gapping out conducting edge modes. Experimentally, such an order is evidenced by the reduction of the conductance at low temperatures [40].

By measuring the spatial dependence of RKKY interaction $[19,42]$, one can obtain information about the chirality and spin polarization of the $\mathrm{QH}$ edge state, which in turn are related to the topological order of the bulk QH liquid [17]. In particular, this technique may be used to detect the nature of the $\mathrm{QH}$ liquid at $v=\frac{5}{2}$ : One can distinguish between a chiral edge state and a nonchiral edge state by confirming whether the spin susceptibility is unidirectional along the edge. One can rule out either a spin-polarized state or a spin-unpolarized state by comparing the in-plane and out-ofplane components of the RKKY interaction, by measuring the spin states in the QDs. For this, one can make use of experimental techniques based on spin-to-charge conversion [2] developed for readout of spin qubits in QDs [43-45]. The numerical values of the scaling exponents also help to identify the true $v=\frac{5}{2}$ state. The advantages of measuring the spin susceptibility are obvious, compared with other approaches detecting topological orders based on edge-bulk correspondence [17], such as measuring the temperature and voltage dependence of quasiparticle tunneling [46]. First, it is easier to vary the sampling point in space than in temperature or voltage, e.g., one may use the setup in Fig. 1 with an array of QDs. Second, information encoded in spin degrees of freedom is more robust than that encoded in charge current, against unfavorable modification due to long-range Coulomb interaction in the device [47]. Compared with electronic FabryPérot [48,49] and Mach-Zehnder [50-52] interferometries, our setup probes the non-Abelian topological orders at $v=\frac{5}{2}$ with a much simpler device geometry and more straightforward data.

The scenario becomes more complicated if one replaces the QDs with quantum antidots [53]. In that case, tunneling of quasiparticles, rather than electrons, defines the coupling between the $\mathrm{QH}$ edge and the antidots. It is still possible to define an RKKY interaction mediated by quasiparticles in the edge state, whose spatial dependence can be used to distinguish different Abelian QH states. For non-Abelian states, however, there are ambiguities in the scaling behavior of the RKKY interaction, arising from the multiple fusion channels of nonAbelian quasiparticles.

To conclude, we have introduced an approach to achieving long-distance entanglement of spin qubits confined in QDs, based on the RKKY interaction mediated by QH edge states. The approach allows for the implementation of quantum gates with long coupling ranges and fast operation times, which would greatly facilitate the development of large-scale quantum computers. From a fundamental point of view, the ability to probe the chirality and the spin polarization of a $\mathrm{QH}$ edge state via measuring the spatial form of the RKKY interaction opens up a new venue for studying electronic and spin physics in $\mathrm{QH}$ systems.

\section{ACKNOWLEDGMENT}

We acknowledge support from the Swiss NSF and NCCR QSIT.

\section{APPENDIX A: EFFECTIVE HAMILTONIAN}

Our starting point is the Hamiltonian in Eq. (1). For weak tunnel coupling between the $\mathrm{QH}$ edge and the QDs, we can treat $H_{\Gamma}=\sum_{i} \Gamma_{i} \mathbf{S}_{i} \cdot \mathbf{I}_{i}$ as a perturbation and make a Schrieffer-Wolff transformation [20,21] to remove terms linear 
in $\Gamma_{i}$ from the Hamiltonian. The transformed Hamiltonian reads as

$$
\bar{H}=e^{S} H e^{-S}=H_{\text {edge }}-\frac{1}{2}\left[\left[H_{\text {edge }}, S\right], S\right]+\ldots,
$$

where $S$ satisfies $\left[H_{\text {edge }}, S\right]=H_{\Gamma}$. Written in terms of the Liouvillian superoperator $\mathcal{L}, S=\mathcal{L}^{-1} H_{\Gamma}$. The leading-order terms in $\Gamma_{i}$ in $\bar{H}$ are given by

$$
\bar{H}_{\Gamma}=-\frac{1}{2}\left[\left[H_{\text {edge }}, S\right], S\right]=\frac{1}{2}\left[\mathcal{L}^{-1} H_{\Gamma}, H_{\Gamma}\right] .
$$

Using $\mathcal{L}^{-1}=-i \int_{0}^{\infty} d t e^{-\eta t} e^{i \mathcal{L} t}$, where $\eta=0^{+}$, we find

$$
\begin{aligned}
\bar{H}_{\Gamma}= & -\frac{i}{2} \int_{0}^{\infty} d t e^{-\eta t}\left[H_{\Gamma}(t), H_{\Gamma}\right] \\
= & -\frac{i}{2} \sum_{i j} \Gamma_{i} \Gamma_{j} \int_{0}^{\infty} d t e^{-\eta t}\left[\mathbf{S}_{i}(t) \cdot \mathbf{I}_{i}, \mathbf{S}_{j}(0) \cdot \mathbf{I}_{j}\right] \\
= & -\frac{1}{2} \int_{0}^{\infty} d t e^{-\eta t}\left\{\sum_{i j} i \Gamma_{i} \Gamma_{j} I_{i}^{\alpha} I_{j}^{\beta}\left[S_{i}^{\alpha}(t), S_{j}^{\beta}(0)\right]\right. \\
& \left.+\sum_{i} \Gamma_{i}^{2} \epsilon^{\alpha \beta \gamma} I_{i}^{\alpha} S_{i}^{\beta}(t) S_{i}^{\gamma}(0)\right\},
\end{aligned}
$$

where we have defined $\hat{O}(t)=e^{i H_{\text {edge }} t} \hat{O} e^{-i H_{\text {edget }} t}$ for an operator $\hat{O}$ and used $\left[I_{i}^{\alpha}, I_{j}^{\beta}\right]=i \delta_{i j} \epsilon^{\alpha \beta \gamma} I_{i}^{\gamma}$, with $\epsilon^{\alpha \beta \gamma}$ the LeviCivita symbol. Summation over repeated spin-component indices (Greek letters) is implied throughout this appendix.

Next, we take the expectation $\langle\ldots\rangle$ over the electronic degrees of freedom in the $\mathrm{QH}$ edge state. This gives an effective Hamiltonian describing the dynamics of localized spins in the adiabatic limit,

$$
H_{\mathrm{eff}}=\left\langle\bar{H}_{\Gamma}\right\rangle=\sum_{i j} \frac{\Gamma_{i} \Gamma_{j}}{2} \chi_{i j}^{\alpha \beta} I_{i}^{\alpha} I_{j}^{\beta}-\sum_{i} B_{i}^{\alpha} I_{i}^{\alpha},
$$

where we have identified the spin susceptibility of conduction electrons

$$
\chi_{i j}^{\alpha \beta}=-i \int_{0}^{\infty} d t e^{-\eta t}\left\langle\left[S_{i}^{\alpha}(t), S_{j}^{\beta}(0)\right]\right\rangle,
$$

and defined effective onsite Zeeman fields for the QDs

$$
B_{i}^{\alpha}=\frac{\Gamma_{i}^{2}}{2} \int_{0}^{\infty} d t e^{-\eta t} \epsilon^{\alpha \beta \gamma}\left\langle S_{i}^{\beta}(t) S_{i}^{\gamma}(0)\right\rangle .
$$

Hermiticity of the Hamiltonian (A4) requires $B_{i}^{\alpha}$ be real, and thus the correlator $\left\langle S_{i}^{\beta}(t) S_{i}^{\gamma}(0)\right\rangle$ should be replaced by $\left\langle\left\{S_{i}^{\beta}(t), S_{i}^{\gamma}(0)\right\}\right\rangle / 2$. We are thus led to the Hamiltonian in Eq. (2).

In deriving the above effective Hamiltonian, we have neglected the external magnetic field $B^{\text {ext }}$ that leads to the formation of the $\mathrm{QH}$ liquid. We now estimate $B_{i}^{\alpha}$ (in units of energy) and compare it with $B^{\text {ext }}$. To this end, we take the continuum limit of Eq. (A6) assuming translation invariance: $B_{i}^{\alpha}=B^{\alpha}(x=a)$, where $a$ is the natural short-distance cutoff, taken as the lattice constant of the host material, and

$$
B^{\alpha}(x)=\frac{l^{2} \Gamma_{i}^{2}}{4} \int_{0}^{\infty} d t e^{-\eta t} \epsilon^{\alpha \beta \gamma}\left\langle\left\{S^{\beta}(x, t), S^{\gamma}(0,0)\right\}\right\rangle,
$$

which in turn can be written as a time-ordered product, such that

$$
B_{i}^{\alpha}=\lim _{x \rightarrow a} \frac{l^{2} \Gamma_{i}^{2}}{2} \int_{0}^{\infty} d t e^{-\eta t} \epsilon^{\alpha \beta \gamma} \operatorname{Re}\left\langle\mathcal{T} S^{\beta}(x, t) S^{\gamma}(0,0)\right\rangle .
$$

In Appendix B, we have obtained the expressions of the spin-density operators $S^{\alpha}(x, t)$ in various $\mathrm{QH}$ states. For spin-unpolarized $\mathrm{QH}$ states we find $S^{x} \propto \cos \left(\phi_{n}+\Delta k x\right)$, $S^{y} \propto \sin \left(\phi_{n}+\Delta k x\right)$, and $S^{z} \propto \partial_{x} \phi_{n}$, where $\phi_{n}$ is a neutral edge mode (defined up to a multiplicative constant). Evaluating the correlators in Eq. (A8), we find $B_{i}^{x}=B_{i}^{y}=0$ and $B_{i}^{z} \sim$ $(\sin \Delta k a) \Gamma_{i}^{2}\left[\chi^{z z}(a)\right]$, where $\left[\chi^{z z}(x)\right]$ is given by Eq. (10). The momentum difference $\Delta k$ depends on the transverse distance between edge modes and can be taken as $\Delta k \sim$ $1 / l_{B}$, where $l_{B} \sim 10 \mathrm{~nm}$ is the magnetic length (the precise evaluation of $\Delta k$ gives a similar result). Using $a=0.565 \mathrm{~nm}$ for GaAs, we perform an estimation similar to that for the effective exchange coupling $\mathcal{J}^{\alpha}$ and find $B_{i}^{z} \simeq 0.06 \mathrm{meV}$. This result is independent of the particular $\mathrm{QH}$ state in consideration. On the other hand, $B^{\text {ext }} \simeq 0.1 \mathrm{meV}$ for typical field strengths of several Tesla in QH liquids. Thus, $B_{i}^{z}$ is in general smaller than or comparable to $B^{\text {ext }}$ in spin-unpolarized states.

For spin-polarized $\mathrm{QH}$ states, applying the assumption $S_{i}^{x}=S_{i}^{y}=0$ yields $B_{i}^{x}=B_{i}^{y}=B_{i}^{z}=0$. In this case, we consider fluctuations in the next order, associated with the next-most-relevant spin operators $\delta S_{i}^{x}, \delta S_{i}^{y}$ in the edge theory. We have $\left\langle\delta S_{i}^{x}\right\rangle=\left\langle\delta S_{i}^{y}\right\rangle=0$. The fluctuations give rise to effective onsite Zeeman fields

$$
\delta B_{i}^{\alpha}=\frac{\Gamma_{i}^{2}}{4} \int_{0}^{\infty} d t e^{-\eta t} \epsilon^{\alpha \beta \gamma}\left\langle\left\{\delta S_{i}^{\beta}(t), \delta S_{i}^{\gamma}(0)\right\}\right\rangle,
$$

which are fully out of plane $\delta B_{i}^{\alpha}=\delta^{\alpha z} \delta B_{i}^{z}$. Simple dimensional analysis shows that the order of magnitude of $\delta S_{i}^{x}, \delta S_{i}^{y}$ differs from those of the (nonvanishing) most relevant spin operators by a factor of $a / \bar{v} \tau$, where $\bar{v}$ is the mean edge velocity and $\tau \sim 1 / E_{F}$ is a typical time scale for the dynamics of conduction electrons. Accordingly, the factor $(a / \bar{v} \tau)^{2}$ enters the relative strength of the effective onsite fields $\left(B_{i}^{z}\right)$ in spin-unpolarized states to those $\left(\delta B_{i}^{z}\right)$ in spin-polarized states (where $B_{i}^{x}=B_{i}^{y}=B_{i}^{z}=0$ ). Our estimation shows that $a / \bar{v} \tau<0.1$, so that $\delta B_{i}^{z} \ll B_{i}^{z}<B^{\text {ext }}$. In the main text we have neglected $\delta B_{i}^{z}$ for simplicity.

In principle, the Zeeman terms $H_{Z}=-\sum_{i} B^{\text {ext }} I_{i}^{z}$ (assuming $\mathbf{B}^{\text {ext }}=B^{\text {ext }} \hat{z}$ ) should be included in the unperturbed Hamiltonian in the Schrieffer-Wolff procedure, i.e., $H_{\text {edge }} \rightarrow$ $H_{\text {edge }}+H_{Z}$ in Eqs. (A1) and (A2) and the definition of time evolution. As a consequence, the first localized-spin operator $I_{i}^{\alpha}$ appearing in the two terms in Eq. (A3) acquires time dependence, in addition to the time dependence in the first conduction-spin operator $S_{i}^{\alpha}$. The dynamics of $I_{i}^{\alpha}$, set by the Zeeman energy $B^{\text {ext }}$, however decouples from that of $S_{i}^{\alpha}$, set by the Fermi energy $E_{F}$, since $E_{F} \gg B^{\text {ext }}$ according to the estimation above. Thus, to a good approximation we 
may neglect the time dependence in $I_{i}^{\alpha}$. We do this for spin-unpolarized states. For spin-polarized states, Eq. (A3) is exact: only the terms with $\alpha=\beta=z$ survive in the equation and we have $I_{i}^{\alpha}(t)=I_{i}^{\alpha}(0)$ since $H_{Z}$ commutes with $I_{i}^{z}$.

We note moreover that $H_{Z}$ also appears in Eq. (A4) for both spin-unpolarized and -polarized QH states. In the main text, we have neglected this term for simplicity. However, $H_{Z}$ must be taken into account for the purpose of implementing two-qubit quantum gates (see Ref. [11] for spin qubits working in perpendicular Zeeman fields).

The effective Hamiltonian in Eq. (A4) describes the system in Fig. 1 in equilibrium. Given a change in the spin state of one of the qubits, the entire electronic system readjusts to achieve new equilibrium. The change of the qubit must be adiabatic in order for the other qubit to sense the change and respond. This means that the switching time $t_{\mathrm{sw}}$ of the first qubit satisfies $t_{\mathrm{sw}} \gg L / \bar{v}$. On the other hand, if the qubit state is changed very fast (nonadiabatically), there will be no effect on the second qubit within time $L / \bar{v}$. In that case, the process is dynamic and is described by the spin susceptibility at finite frequencies. For $L=1 \mu \mathrm{m}, L / \bar{v} \simeq 10 \mathrm{ps,}$ which is much shorter than the ideal gate-operation time $t_{\mathrm{sw}} \simeq 1 \mathrm{~ns}$. Thus, the requirement for adiabaticity does not place much restriction on the operation of spin qubits.

\section{APPENDIX B: SPIN SUSCEPTIBILITY}

In this appendix, we calculate the spin susceptibility for the $\mathrm{QH}$ states listed in Table I. The formula is given by Eq. (4). First, we compute the correlators in the zero-temperature limit

$$
\mathcal{G}^{\alpha}(x, t)=\left\langle\mathcal{T} S^{\alpha}(x, t) S^{\alpha}(0,0)\right\rangle,
$$

where $\alpha=x, y, z$. We focus on the scaling behaviors of these correlators and neglect the proportionality constants. Next, we evaluate the time integral

$$
\chi^{\alpha \alpha}(x)=2 l^{2} \int_{0}^{\infty} d t e^{-\eta t} \operatorname{Im} \mathcal{G}^{\alpha}(x, t),
$$

where $\eta=0^{+}$. Restoring the proportionality constants, we obtain the full expression of the spin susceptibility.

\section{Correlators}

\section{a. Laughlin states at $v=1 / m$}

The Lagrangian density that describes the edge state of the $v=1 / m$ ( $m$ is an odd integer) Laughlin state is

$$
L=\frac{m}{4 \pi}\left[\partial_{t} \phi \partial_{x} \phi-v\left(\partial_{x} \phi\right)^{2}\right],
$$

where $v$ is the velocity of the edge mode described by bosonic field $\phi$. We assume the edge state is left moving. Electrons in the edge state are described by the vertex operator $\psi=$ $\frac{1}{\sqrt{2 \pi a}} e^{-i k_{F} x} e^{-i m \phi}$, where $a$ is the short-distance cutoff and $k_{F}$ is the Fermi momentum. Here and throughout this appendix, we omit the Klein factors in the electron operators, which will drop out when evaluating the average. Since the edge state is spin polarized, all the electrons have the same spin $\sigma$. Let us assume $\sigma=\uparrow$. Using Eq. (5) and neglecting transitions to higher Landau levels, we find $S^{x}=S^{y}=0$, and

$$
S^{z}=\frac{1}{2} \psi^{\dagger} \psi \propto \partial_{x} \phi .
$$

The correlator of $\phi$ can be read from Eq. (B3), $\langle\mathcal{T} \phi(x, t) \phi(0,0)\rangle=-v \ln (x+v t-i \delta)+$ const, where $\delta$ is defined as a positive infinitesimal throughout the appendix. This gives

$$
\mathcal{G}^{z}(x, t) \propto \frac{v}{(x+v t-i \delta)^{2}},
$$

whereas $\mathcal{G}^{x}=\mathcal{G}^{y}=0$. Substituting Eq. (B5) in Eq. (B2) we obtain the spin susceptibility in Laughlin states.

\section{b. $Q H$ state at $v=2$}

The $v=2 \mathrm{QH}$ state has two bosonic edge modes $\phi_{\uparrow}, \phi_{\downarrow}$, propagating in the same direction, where $\phi_{\uparrow}$ has spin up and $\phi_{\downarrow}$ has spin down. The Lagrangian density is

$$
L=\frac{1}{4 \pi}\left\{\sum_{i=\uparrow, \downarrow}\left[\partial_{t} \phi_{i} \partial_{x} \phi_{i}-v_{i}\left(\partial_{x} \phi_{i}\right)^{2}\right]-2 u \partial_{x} \phi_{\uparrow} \partial_{x} \phi_{\downarrow}\right\},
$$

where $v_{i}$ is the velocity of $\phi_{i}$ and $u>0$ is the repulsive Coulomb interaction between $\phi_{\uparrow}$ and $\phi_{\downarrow}$. We assume the edge modes are left moving. The most relevant electron operators are $\psi_{i}=\frac{1}{\sqrt{2 \pi a}} e^{-i k_{F, i} x} e^{-i \phi_{i}}$, where $k_{F, i}$ is the Fermi momentum of $\phi_{i}$. The spin-density operators are

$$
\begin{aligned}
& S^{x}=\frac{1}{2}\left(\psi_{\uparrow}^{\dagger} \psi_{\downarrow}+\text { H.c. }\right) \propto e^{i \Delta k x} e^{i\left(\phi_{\uparrow}-\phi_{\downarrow}\right)}+\text { H.c. }, \\
& S^{y}=\frac{1}{2}\left(-i \psi_{\uparrow}^{\dagger} \psi_{\downarrow}+\text { H.c. }\right) \propto-i e^{i \Delta k x} e^{i\left(\phi_{\uparrow}-\phi_{\downarrow}\right)}+\text { H.c. }, \\
& S^{z}=\frac{1}{2}\left(\psi_{\uparrow}^{\dagger} \psi_{\uparrow}-\psi_{\downarrow}^{\dagger} \psi_{\downarrow}\right) \propto \partial_{x}\left(\phi_{\uparrow}-\phi_{\downarrow}\right),
\end{aligned}
$$

where $\Delta k=k_{F, \uparrow}-k_{F, \downarrow}$ is the gauge-invariant momentum difference, proportional to the magnetic flux penetrating between the two edge modes.

To compute the correlators, we define eigenmodes

$$
\begin{aligned}
& \phi_{+}=\cos \varphi \phi_{\uparrow}+\sin \varphi \phi_{\downarrow}, \\
& \phi_{-}=-\sin \varphi \phi_{\uparrow}+\cos \varphi \phi_{\downarrow},
\end{aligned}
$$

where $\tan 2 \varphi=\frac{2 u}{v_{\uparrow}-v_{\downarrow}}$, which diagonalize the edge theory

$$
L=\frac{1}{4 \pi} \sum_{i=+,-}\left[\partial_{t} \phi_{i} \partial_{x} \phi_{i}-v_{i}\left(\partial_{x} \phi_{i}\right)^{2}\right]
$$

where $v_{ \pm}=\frac{1}{2}\left[v_{\uparrow}+v_{\downarrow} \pm \sqrt{\left(v_{\uparrow}-v_{\downarrow}\right)^{2}+4 u^{2}}\right]$. According to the experiment [25], $v_{+} \gg v_{-}$as a result of the strong Coulomb interaction $u$. Expressing the spin-density operators in eigenmodes, it is straightforward to obtain

$$
\begin{aligned}
& \mathcal{G}^{x}(x, t) \propto \cos (\Delta k x)\left[\frac{1}{x+v_{+} t-i \delta}\right]^{c_{+}^{2}}\left[\frac{1}{x+v_{-} t-i \delta}\right]^{c_{-}^{2}}, \\
& \mathcal{G}^{y}(x, t) \propto \cos (\Delta k x)\left[\frac{1}{x+v_{+} t-i \delta}\right]^{c_{+}^{2}}\left[\frac{1}{x+v_{-} t-i \delta}\right]^{c_{-}^{2}}, \\
& \mathcal{G}^{z}(x, t) \propto \frac{c_{+}^{2}}{\left(x+v_{+} t-i \delta\right)^{2}}+\frac{c_{-}^{2}}{\left(x+v_{-} t-i \delta\right)^{2}},
\end{aligned}
$$


where the functions $c_{ \pm}(\varphi)=\cos \varphi \mp \sin \varphi$. Notice that $c_{+}^{2}(\varphi)+c_{-}^{2}(\varphi)=2$, i.e., the scaling exponents of the correlators are integral invariant, independent of the angle $\varphi$ which depends on the interedge interaction. This is a well-known property of chiral QH edge states [17].

\section{c. $Q H$ state at $v=\frac{2}{3}$}

The $v=\frac{2}{3} \mathrm{QH}$ state can be spin unpolarized at low fields and spin polarized at high fields [29]. We first consider the spinunpolarized state. It has two bosonic edge modes $\phi_{\uparrow}$ and $\phi_{\downarrow}$, where $\phi_{\uparrow}$ has spin up and $\phi_{\downarrow}$ has spin down. The Lagrangian density is

$$
L=\frac{1}{4 \pi} \sum_{i, j=\uparrow, \downarrow}\left[K_{i j} \partial_{t} \phi_{i} \partial_{x} \phi_{j}-V_{i j} \partial_{x} \phi_{i} \partial_{x} \phi_{j}\right],
$$

where

$$
K=\left(\begin{array}{ll}
1 & 2 \\
2 & 1
\end{array}\right) \quad \text { and } \quad V=\left(\begin{array}{cc}
v_{\uparrow} & u \\
u & v_{\downarrow}
\end{array}\right),
$$

with $v_{i}$ the velocity of $\phi_{i}$ and $u$ the interedge interaction. The eigenvalues of the $K$ matrix have opposite signs, so the edge state is nonchiral.

Experiment [26] revealed that the $v=\frac{2}{3}$ edge state consists of a charged mode and a neutral mode, moving in opposite directions. To connect the parameters in the edge theory described by Eq. (B11) with experiment, we change to the physical basis of charged mode $\phi_{\rho}=\phi_{\uparrow}+\phi_{\downarrow}$ and neutral mode $\phi_{n}=\phi_{\uparrow}-\phi_{\downarrow}$ :

$$
\begin{aligned}
L= & \frac{1}{4 \pi}\left[\frac{3}{2} \partial_{t} \phi_{\rho} \partial_{x} \phi_{\rho}-\frac{1}{2} \partial_{t} \phi_{n} \partial_{x} \phi_{n}-\frac{3}{2} v_{\rho}\left(\partial_{x} \phi_{\rho}\right)^{2}\right. \\
& \left.-\frac{1}{2} v_{n}\left(\partial_{x} \phi_{n}\right)^{2}-2 v_{\rho n} \partial_{x} \phi_{\rho} \partial_{x} \phi_{n}\right],
\end{aligned}
$$

where $v_{\rho}=\frac{1}{3}\left(\frac{v_{\uparrow}}{2}+\frac{v_{\downarrow}}{2}+u\right), v_{n}=\frac{v_{\uparrow}}{2}+\frac{v_{\downarrow}}{2}-u$, and $v_{\rho n}=$ $\frac{1}{4}\left(v_{\uparrow}-v_{\downarrow}\right)$. In general, $v_{\uparrow} \neq v_{\downarrow}$ due to finite Zeeman splitting. The charged-mode velocity $v_{\rho}$, determined by the large Coulomb energy scale, is expected to be much greater in order of magnitude than the neutral-mode velocity $v_{n}$ and the interaction $v_{\rho n}$. We therefore assume $v_{\rho} \gg v_{n} \sim v_{\rho n}$. In particular, we assume that the scaling dimensions of quasiparticle operators in the real case do not deviate much from those in the case $v_{\rho n}=0$. With this assumption, we can determine the most relevant electron operators in the edge theory, which are $\psi_{\uparrow} \propto e^{-i\left(2 k_{F, \uparrow}+k_{F, \downarrow}\right) x} e^{-i\left(2 \phi_{\uparrow}+\phi_{\downarrow}\right)}$, with spin up, and $\psi_{\downarrow} \propto e^{-i\left(k_{F, \uparrow}+2 k_{F, \downarrow}\right) x} e^{-i\left(\phi_{\uparrow}+2 \phi_{\downarrow}\right)}$, with spin down, where $k_{F, \uparrow}$ and $k_{F, \downarrow}$ are momentumlike constants related to the spatial locations of the edge modes $\phi_{\uparrow}$ and $\phi_{\downarrow}$. The spin-density operators are obtained by computing the operator product expansions (OPEs) of the electron operators and keeping the most singular terms. We find

$$
\begin{aligned}
& S^{x}=\frac{1}{2}\left(\psi_{\uparrow}^{\dagger} \psi_{\downarrow}+\text { H.c. }\right) \propto e^{i \Delta k x} e^{i \phi_{n}}+\text { H.c., } \\
& S^{y}=\frac{1}{2}\left(-i \psi_{\uparrow}^{\dagger} \psi_{\downarrow}+\text { H.c. }\right) \propto-i e^{i \Delta k x} e^{i \phi_{n}}+\text { H.c. }, \\
& S^{z}=\frac{1}{2}\left(\psi_{\uparrow}^{\dagger} \psi_{\uparrow}-\psi_{\downarrow}^{\dagger} \psi_{\downarrow}\right) \propto \partial_{x} \phi_{n},
\end{aligned}
$$

where $\Delta k=k_{F, \uparrow}-k_{F, \downarrow}$.
In terms of eigenmodes

$$
\begin{aligned}
& \phi_{+}=\sqrt{\frac{3}{2}} \cosh \theta \phi_{\rho}+\sqrt{\frac{1}{2}} \sinh \theta \phi_{n}, \\
& \phi_{-}=\sqrt{\frac{3}{2}} \sinh \theta \phi_{\rho}+\sqrt{\frac{1}{2}} \cosh \theta \phi_{n},
\end{aligned}
$$

where $\tanh 2 \theta=\frac{4}{\sqrt{3}} \frac{v_{\rho n}}{v_{\rho}+v_{n}}$, the edge theory is diagonalized,

$$
L=\frac{1}{4 \pi}\left[\partial_{t} \phi_{+} \partial_{x} \phi_{+}-\partial_{t} \phi_{-} \partial_{x} \phi_{-}-\sum_{i=+,-} v_{i}\left(\partial_{x} \phi_{i}\right)^{2}\right],
$$

where $\quad v_{+}=\frac{1}{\cosh 2 \theta}\left(\cosh ^{2} \theta v_{\rho}-\sinh ^{2} \theta v_{n}\right)$ and $v_{-}=$ $\frac{1}{\cosh 2 \theta}\left(\cosh ^{2} \theta v_{n}-\sinh ^{2} \theta v_{\rho}\right)$. Since $v_{\rho} \gg v_{n} \sim v_{\rho n}$, we have $\theta \ll 1$ and thus $v_{+} \simeq v_{\rho}, v_{-} \simeq v_{n}$, and $v_{+} \gg v_{-}$. The correlators are evaluated to be

$$
\begin{aligned}
& \mathcal{G}^{x}(x, t) \propto \cos (\Delta k x)\left[\frac{1}{x+v_{+} t-i \delta}\right]^{\tilde{c}_{+}^{2}}\left[\frac{1}{x-v_{-} t+i \delta}\right]^{\tilde{c}_{-}^{2}}, \\
& \mathcal{G}^{y}(x, t) \propto \cos (\Delta k x)\left[\frac{1}{x+v_{+} t-i \delta}\right]^{\tilde{c}_{+}^{2}}\left[\frac{1}{x-v_{-} t+i \delta}\right]^{\tilde{c}_{-}^{2}}, \\
& \mathcal{G}^{z}(x, t) \propto \frac{\tilde{c}_{+}^{2}}{\left(x+v_{+} t-i \delta\right)^{2}}+\frac{\tilde{c}_{-}^{2}}{\left(x-v_{-} t+i \delta\right)^{2}},
\end{aligned}
$$

where the functions $\tilde{c}_{+}(\theta)=\sqrt{2} \sinh \theta$ and $\tilde{c}_{-}(\theta)=$ $\sqrt{2} \cosh \theta$. Notice that $\tilde{c}_{+}^{2}(\theta)+\tilde{c}_{-}^{2}(\theta)=2\left(1+2 \sinh ^{2} \theta\right)$, i.e., the scaling exponents are nonuniversal and depend on the parameters in the Hamiltonian, through $\theta$. This reflects the nonchiral nature of the edge state.

Next, we discuss the spin-polarized state at $v=\frac{2}{3}$. It has two bosonic edge modes $\phi_{1}$ and $\phi_{2}$, having the same spin polarization (assuming they are spin up). The Lagrangian density has the same form of Eq. (B11), with

$$
K=\left(\begin{array}{cc}
1 & 0 \\
0 & -3
\end{array}\right) \text { and } V=\left(\begin{array}{cc}
v_{1} & u^{\prime} \\
u^{\prime} & 3 v_{2}
\end{array}\right)
$$

This is also a nonchiral state. The charged mode and the neutral mode in the edge theory are identified as $\phi_{\rho}=\phi_{1}+\phi_{2}$ and $\phi_{n}=\phi_{1}+3 \phi_{2}$, respectively, in terms of which the Lagrangian density recovers the expression in Eq. (B13), with $v_{\rho}=\frac{3}{2} v_{1}+$ $\frac{1}{2} v_{2}-u^{\prime}, v_{n}=\frac{1}{2} v_{1}+\frac{3}{2} v_{2}-u^{\prime}$, and $v_{\rho n}=-\frac{3}{4} v_{1}-\frac{3}{4} v_{2}+u^{\prime}$. Again, we assume $v_{\rho} \gg v_{n} \sim v_{\rho n}$. The most relevant electron operators are $\psi_{1} \propto e^{-i\left(2 k_{F, 1}+3 k_{F, 2}\right) x} e^{-i\left(2 \phi_{1}+3 \phi_{2}\right)}$ and $\psi_{2} \propto$ $e^{-i k_{F, 1} x} e^{-i \phi_{1}}$, both with spin up, where $k_{F, 1}$ and $k_{F, 2}$ are constants. Using Eq. (5) and OPE, we find $S^{x}=S^{y}=0$ and $S^{z}=S_{f}^{z}+S_{b}^{z}$, where

$$
\begin{aligned}
& S_{f}^{z}=\frac{1}{2}\left(\psi_{1}^{\dagger} \psi_{1}+\psi_{2}^{\dagger} \psi_{2}\right) \propto \partial_{x} \phi_{\rho}, \\
& S_{b}^{z}=\frac{1}{2}\left(\psi_{1}^{\dagger} \psi_{2}+\text { H.c. }\right) \propto e^{i \Delta k x} e^{i \phi_{n}}+\text { H.c. },
\end{aligned}
$$

where $\Delta k=k_{F, 1}+3 k_{F, 2}$ is interpreted as the Fermimomentum difference between the elementary edge modes $\phi_{1}$ and $\phi_{2}$. The rest of the analysis resembles that for the spin-unpolarized state. We diagonalize the edge theory using the free fields $\phi_{+}, \phi_{-}$defined in Eq. (B15) and evaluate the 
correlators. We find $\mathcal{G}^{x}=\mathcal{G}^{y}=0$ and $\mathcal{G}^{z}=\mathcal{G}_{f}^{z}+\mathcal{G}_{b}^{z}$, where

$$
\begin{aligned}
\mathcal{G}_{f}^{z}(x, t) & \propto \frac{\tilde{c}_{-}^{2}}{\left(x+v_{+} t-i \delta\right)^{2}}+\frac{\tilde{c}_{+}^{2}}{\left(x-v_{-} t+i \delta\right)^{2}}, \\
\mathcal{G}_{b}^{z}(x, t) & \propto \cos (\Delta k x)\left[\frac{1}{x+v_{+} t-i \delta}\right]^{\tilde{c}_{+}^{2}}\left[\frac{1}{x-v_{-} t+i \delta}\right]^{\tilde{c}_{-}^{2}} .
\end{aligned}
$$

\section{d. 331 state at $v=\frac{5}{2}$}

We now turn to the $\mathrm{QH}$ state at $v=\frac{5}{2}$. This $\mathrm{QH}$ state is usually modeled by combining a $v=2$ integer $\mathrm{QH}$ state in the lowest Landau level, which is treated as an inert background assuming no Landau level mixing, and a $v=\frac{1}{2}$ fractional $\mathrm{QH}$ state in the second Landau level, which is assumed to capture the full topological order of the $\mathrm{QH}$ liquid. We study the RKKY interaction mediated solely by the fractional edge state. In the following, we consider several topological orders proposed for the fractional edge state, including Halperin's 331 and 113 states [30,31], the Pfaffian state [32], the anti-Pfaffian state $[33,34]$ and the $\mathrm{SU}(2)_{2}$ state [35]. Motivated by the experiment [26], we will always assume separation of charged and neutral degrees of freedom in the edge state. Moreover, we assume that the charged-mode velocity is much greater than other physical parameters, by a similar argument to that for the $\mathrm{QH}$ state at $v=\frac{2}{3}$.

We start from Halperin's 331 state, which has a spinunpolarized version and a spin-polarized version. The Lagrangian density for the edge of the spin-unpolarized 331 state has the same form of Eq. (B11), with

$$
K=\left(\begin{array}{ll}
3 & 1 \\
1 & 3
\end{array}\right) \quad \text { and } \quad V=\left(\begin{array}{cc}
v_{\uparrow} & u \\
u & v_{\downarrow}
\end{array}\right),
$$

where $v_{\uparrow}$ and $v_{\downarrow}$ are the velocities of edge modes $\phi_{\uparrow}$ and $\phi_{\downarrow}$, respectively, and $u$ the interedge interaction. Here, $\phi_{\uparrow}$ is a spin-up mode and $\phi_{\downarrow}$ is a spin-down mode. The 331 state is chiral. The physical charged mode and neutral mode are defined as $\phi_{\rho}=\phi_{\uparrow}+\phi_{\downarrow}$ and $\phi_{n}=\phi_{\uparrow}-\phi_{\downarrow}$, respectively, in terms of which the Lagrangian density is

$$
\begin{aligned}
L= & \frac{1}{4 \pi}\left[2 \partial_{t} \phi_{\rho} \partial_{x} \phi_{\rho}+\partial_{t} \phi_{n} \partial_{x} \phi_{n}-2 v_{\rho}\left(\partial_{x} \phi_{\rho}\right)^{2}\right. \\
& \left.-v_{n}\left(\partial_{x} \phi_{n}\right)^{2}-2 v_{\rho n} \partial_{x} \phi_{\rho} \partial_{x} \phi_{n}\right],
\end{aligned}
$$

where $v_{\rho}=\frac{v_{\uparrow}}{8}+\frac{v_{\downarrow}}{8}+\frac{u}{4}, \quad v_{n}=\frac{v_{\uparrow}}{4}+\frac{v_{\downarrow}}{4}-\frac{u}{2}, \quad$ and $\quad v_{\rho n}=$ $\frac{1}{4}\left(v_{\uparrow}-v_{\downarrow}\right)$. Assuming $v_{\rho} \gg v_{n} \sim v_{\rho n}$, the most relevant electron operators are $\psi_{\uparrow} \propto e^{-i\left(3 k_{F, \uparrow}+k_{F, \downarrow}\right) x} e^{-i\left(3 \phi_{\uparrow}+\phi_{\downarrow}\right)}$, with spin up, and $\psi_{\downarrow} \propto e^{-i\left(k_{F, \uparrow}+3 k_{F, \downarrow}\right) x} e^{-i\left(\phi_{\uparrow}+3 \phi_{\downarrow}\right)}$, with spin down, where $k_{F, \uparrow}$ and $k_{F, \downarrow}$ are constants. The spin-density operators are

$$
\begin{aligned}
& S^{x}=\frac{1}{2}\left(\psi_{\uparrow}^{\dagger} \psi_{\downarrow}+\text { H.c. }\right) \propto e^{i \Delta k x} e^{i 2 \phi_{n}}+\text { H.c., } \\
& S^{y}=\frac{1}{2}\left(-i \psi_{\uparrow}^{\dagger} \psi_{\downarrow}+\text { H.c. }\right) \propto-i e^{i \Delta k x} e^{i 2 \phi_{n}}+\text { H.c. }, \\
& S^{z}=\frac{1}{2}\left(\psi_{\uparrow}^{\dagger} \psi_{\uparrow}-\psi_{\downarrow}^{\dagger} \psi_{\downarrow}\right) \propto \partial_{x} \phi_{n},
\end{aligned}
$$

where $\Delta k=2 k_{F, \uparrow}-2 k_{F, \downarrow}$. To evaluate the correlators of $S^{\alpha}$, we define eigenmodes

$$
\begin{aligned}
& \phi_{+}=\sqrt{2} \cos \theta \phi_{\rho}+\sin \theta \phi_{n}, \\
& \phi_{-}=-\sqrt{2} \sin \theta \phi_{\rho}+\cos \theta \phi_{n},
\end{aligned}
$$

where $\tan 2 \theta=\frac{\sqrt{2} v_{\rho n}}{v_{\rho}-v_{n}}$. We have $\theta \ll 1$. We find

$$
\begin{aligned}
& \mathcal{G}^{x}(x, t) \propto \cos (\Delta k x)\left[\frac{1}{x+v_{+} t-i \delta}\right]^{\bar{c}_{+}^{2}}\left[\frac{1}{x+v_{-} t-i \delta}\right]^{\bar{c}_{-}^{2}}, \\
& \mathcal{G}^{y}(x, t) \propto \cos (\Delta k x)\left[\frac{1}{x+v_{+} t-i \delta}\right]^{\bar{c}_{+}^{2}}\left[\frac{1}{x+v_{-} t-i \delta}\right]^{\bar{c}_{-}^{2}}, \\
& \mathcal{G}^{z}(x, t) \propto \frac{\bar{c}_{+}^{2}}{\left(x+v_{+} t-i \delta\right)^{2}}+\frac{\bar{c}_{-}^{2}}{\left(x+v_{-} t-i \delta\right)^{2}},
\end{aligned}
$$

where $\bar{c}_{+}(\theta)=2 \sin \theta$ and $\bar{c}_{-}(\theta)=2 \cos \theta$. The parameters $v_{+}$ and $v_{-}$are the velocities of $\phi_{+}$and $\phi_{-}$, respectively. We have $v_{+} \simeq v_{\rho}, v_{-} \simeq v_{n}$, and $v_{+} \gg v_{-}$.

The spin-polarized 331 state has two bosonic edge modes $\phi_{1}$ and $\phi_{2}$, having the same spin polarization (assuming they are spin up). The Lagrangian density has the same form of Eq. (B11), with

$$
K=\left(\begin{array}{cc}
3 & -2 \\
-2 & 4
\end{array}\right) \text { and } V=\left(\begin{array}{ll}
v_{1} & u^{\prime} \\
u^{\prime} & v_{2}
\end{array}\right)
$$

The physical charged and neutral modes are identified as $\phi_{\rho}=$ $\phi_{1}$ and $\phi_{n}=-\phi_{1}+2 \phi_{2}$, respectively, in terms of which the Lagrangian density recovers the form in Eq. (B22), with $v_{\rho}=$ $\frac{1}{2} v_{1}+\frac{1}{8} v_{2}+\frac{1}{2} u^{\prime}, v_{n}=\frac{1}{4} v_{2}$, and $v_{\rho n}=\frac{1}{4} v_{2}+\frac{1}{2} u^{\prime}$. Assuming $v_{\rho} \gg v_{n} \sim v_{\rho n}$, the most relevant electron operators are $\psi_{1} \propto$ $e^{-i\left(k_{F, 1}+2 k_{F, 2}\right) x} e^{-i\left(\phi_{1}+2 \phi_{2}\right)}$ and $\psi_{2} \propto e^{-i\left(3 k_{F, 1}-2 k_{F, 2}\right) x} e^{-i\left(3 \phi_{1}-2 \phi_{2}\right)}$ both with spin up, where $k_{F, 1}$ and $k_{F, 2}$ are constants. The spindensity operators are $S^{x}=S^{y}=0$ and $S^{z}=S_{f}^{z}+S_{b}^{z}$, where

$$
\begin{aligned}
& S_{f}^{z}=\frac{1}{2}\left(\psi_{1}^{\dagger} \psi_{1}+\psi_{2}^{\dagger} \psi_{2}\right) \propto \partial_{x} \phi_{\rho}, \\
& S_{b}^{z}=\frac{1}{2}\left(\psi_{1}^{\dagger} \psi_{2}+\text { H.c. }\right) \propto e^{i \Delta k x} e^{i 2 \phi_{n}}+\text { H.c. },
\end{aligned}
$$

with $\Delta k=-2 k_{F, 1}+4 k_{F, 2}$. Using the definition of eigenmodes in Eq. (B24), we find $\mathcal{G}^{x}=\mathcal{G}^{y}=0$ and $\mathcal{G}^{z}=\mathcal{G}_{f}^{z}+\mathcal{G}_{b}^{z}$, where

$$
\begin{gathered}
\mathcal{G}_{f}^{z}(x, t) \propto \frac{\bar{c}_{-}^{2}}{\left(x+v_{+} t-i \delta\right)^{2}}+\frac{\bar{c}_{+}^{2}}{\left(x+v_{-} t-i \delta\right)^{2}}, \\
\mathcal{G}_{b}^{z}(x, t) \propto \cos (\Delta k x)\left[\frac{1}{x+v_{+} t-i \delta}\right]^{\bar{c}_{+}^{2}}\left[\frac{1}{x+v_{-} t-i \delta}\right]^{\bar{c}_{-}^{2}} . \\
\text { e. } 113 \text { state at } v=\frac{5}{2}
\end{gathered}
$$

The 113 state also has a spin-unpolarized version and a spinpolarized version. The edge theory of the spin-unpolarized 113 state is of the form of Eq. (B11), with

$$
K=\left(\begin{array}{ll}
1 & 3 \\
3 & 1
\end{array}\right) \quad \text { and } \quad V=\left(\begin{array}{cc}
v_{\uparrow} & u \\
u & v_{\downarrow}
\end{array}\right)
$$

where $v_{\uparrow}$ and $v_{\downarrow}$ are the velocities of edge modes $\phi_{\uparrow}$ and $\phi_{\downarrow}$, respectively, and $u$ the interedge interaction. Here, $\phi_{\uparrow}$ is a spin-up mode and $\phi_{\downarrow}$ is a spin-down mode. The 113 state is nonchiral. Switching to the physical basis of charged 
mode $\phi_{\rho}=\phi_{\uparrow}+\phi_{\downarrow}$ and neutral mode $\phi_{n}=\phi_{\uparrow}-\phi_{\downarrow}$, the Lagrangian density becomes

$$
\begin{aligned}
L= & \frac{1}{4 \pi}\left[2 \partial_{t} \phi_{\rho} \partial_{x} \phi_{\rho}-\partial_{t} \phi_{n} \partial_{x} \phi_{n}-2 v_{\rho}\left(\partial_{x} \phi_{\rho}\right)^{2}\right. \\
& \left.-v_{n}\left(\partial_{x} \phi_{n}\right)^{2}-2 v_{\rho n} \partial_{x} \phi_{\rho} \partial_{x} \phi_{n}\right],
\end{aligned}
$$

where $v_{\rho}=\frac{v_{\uparrow}}{8}+\frac{v_{\downarrow}}{8}+\frac{u}{4}, \quad v_{n}=\frac{v_{\uparrow}}{4}+\frac{v_{\downarrow}}{4}-\frac{u}{2}, \quad$ and $\quad v_{\rho n}=$ $\frac{1}{4}\left(v_{\uparrow}-v_{\downarrow}\right)$. Assuming $v_{\rho} \gg v_{n} \sim v_{\rho n}$, the most relevant electron operators are $\psi_{\uparrow} \propto e^{-i\left(3 k_{F, \uparrow}+k_{F, \downarrow}\right) x} e^{-i\left(3 \phi_{\uparrow}+\phi_{\downarrow}\right)}$, with spin up, and $\psi_{\downarrow} \propto e^{-i\left(k_{F, \uparrow}+3 k_{F, \downarrow}\right) x} e^{-i\left(\phi_{\uparrow}+3 \phi_{\downarrow}\right)}$, with spin down, where $k_{F, \uparrow}$ and $k_{F, \downarrow}$ are constants. The spin-density operators are

$$
\begin{aligned}
& S^{x}=\frac{1}{2}\left(\psi_{\uparrow}^{\dagger} \psi_{\downarrow}+\text { H.c. }\right) \propto e^{i \Delta k x} e^{i 2 \phi_{n}}+\text { H.c. } \\
& S^{y}=\frac{1}{2}\left(-i \psi_{\uparrow}^{\dagger} \psi_{\downarrow}+\text { H.c. }\right) \propto-i e^{i \Delta k x} e^{i 2 \phi_{n}}+\text { H.c. }, \\
& S^{z}=\frac{1}{2}\left(\psi_{\uparrow}^{\dagger} \psi_{\uparrow}-\psi_{\downarrow}^{\dagger} \psi_{\downarrow}\right) \propto \partial_{x} \phi_{n},
\end{aligned}
$$

where $\Delta k=2 k_{F, \uparrow}-2 k_{F, \downarrow}$. The eigenmodes are defined as

$$
\begin{aligned}
& \phi_{+}=\sqrt{2} \cosh \theta \phi_{\rho}+\sinh \theta \phi_{n}, \\
& \phi_{-}=\sqrt{2} \sinh \theta \phi_{\rho}+\cosh \theta \phi_{n},
\end{aligned}
$$

where $\tanh 2 \theta=\frac{\sqrt{2} v_{\rho n}}{v_{\rho}+v_{n}}$. We have $\theta \ll 1$. We find

$$
\begin{aligned}
& \mathcal{G}^{x}(x, t) \propto \cos (\Delta k x)\left[\frac{1}{x+v_{+} t-i \delta}\right]^{2 \tilde{c}_{+}^{2}}\left[\frac{1}{x-v_{-} t+i \delta}\right]^{2 \tilde{c}_{-}^{2}}, \\
& \mathcal{G}^{y}(x, t) \propto \cos (\Delta k x)\left[\frac{1}{x+v_{+} t-i \delta}\right]^{2 \tilde{c}_{+}^{2}}\left[\frac{1}{x-v_{-} t+i \delta}\right]^{2 \tilde{c}_{-}^{2}}, \\
& \mathcal{G}^{z}(x, t) \propto \frac{\tilde{c}_{+}^{2}}{\left(x+v_{+} t-i \delta\right)^{2}}+\frac{\tilde{c}_{-}^{2}}{\left(x-v_{-} t+i \delta\right)^{2}},
\end{aligned}
$$

where $\tilde{c}_{+}(\theta)=\sqrt{2} \sinh \theta$ and $\tilde{c}_{-}(\theta)=\sqrt{2} \cosh \theta$. The parameters $v_{+}$and $v_{-}$are the velocities of $\phi_{+}$and $\phi_{-}$, respectively. We have $v_{+} \simeq v_{\rho}, v_{-} \simeq v_{n}$, and $v_{+} \gg v_{-}$.

The spin-polarized 113 state has two bosonic edge modes $\phi_{1}$ and $\phi_{2}$, having the same spin polarization (assume they are spin up). The Lagrangian density has the same form of Eq. (B11), with

$$
K=\left(\begin{array}{rr}
1 & 2 \\
2 & -4
\end{array}\right) \quad \text { and } \quad V=\left(\begin{array}{ll}
v_{1} & u^{\prime} \\
u^{\prime} & v_{2}
\end{array}\right)
$$

The charged and the neutral modes are $\phi_{\rho}=\phi_{1}$ and $\phi_{n}=$ $-\phi_{1}+2 \phi_{2}$, respectively, in terms of which the Lagrangian density recovers the form in Eq. (B30), with $v_{\rho}=\frac{1}{2} v_{1}+$ $\frac{1}{8} v_{2}+\frac{1}{2} u^{\prime}, v_{n}=\frac{1}{4} v_{2}$, and $v_{\rho n}=\frac{1}{4} v_{2}+\frac{1}{2} u^{\prime}$. Assuming $v_{\rho} \gg$ $v_{n} \sim v_{\rho n}$, the most relevant electron operators are $\psi_{1} \propto$ $e^{-i\left(k_{F, 1}+2 k_{F, 2}\right) x} e^{-i\left(\phi_{1}+2 \phi_{2}\right)}$ and $\psi_{2} \propto e^{-i\left(3 k_{F, 1}-2 k_{F, 2}\right) x} e^{-i\left(3 \phi_{1}-2 \phi_{2}\right)}$, both with spin up, where $k_{F, 1}$ and $k_{F, 2}$ are constants. The spin-density operators are $S^{x}=S^{y}=0$ and $S^{z}=S_{f}^{z}+S_{b}^{z}$, where

$$
\begin{aligned}
& S_{f}^{z}=\frac{1}{2}\left(\psi_{1}^{\dagger} \psi_{1}+\psi_{2}^{\dagger} \psi_{2}\right) \propto \partial_{x} \phi_{\rho} \\
& S_{b}^{z}=\frac{1}{2}\left(\psi_{1}^{\dagger} \psi_{2}+\text { H.c. }\right) \propto e^{i \Delta k x} e^{i 2 \phi_{n}}+\text { H.c. },
\end{aligned}
$$

with $\Delta k=-2 k_{F, 1}+4 k_{F, 2}$. Using the definition of eigenmodes in Eq. (B32), we find $\mathcal{G}^{x}=\mathcal{G}^{y}=0$ and $\mathcal{G}^{z}=\mathcal{G}_{f}^{z}+\mathcal{G}_{b}^{z}$, where

$$
\begin{aligned}
& \mathcal{G}_{f}^{z}(x, t) \propto \frac{\tilde{c}_{-}^{2}}{\left(x+v_{+} t-i \delta\right)^{2}}+\frac{\tilde{c}_{+}^{2}}{\left(x-v_{-} t+i \delta\right)^{2}}, \\
& \mathcal{G}_{b}^{z}(x, t) \propto \cos (\Delta k x)\left[\frac{1}{x+v_{+} t-i \delta}\right]^{2 \tilde{c}_{+}^{2}}\left[\frac{1}{x-v_{-} t+i \delta}\right]^{2 \tilde{c}_{-}^{2}} .
\end{aligned}
$$

\section{f. Pfaffian state at $v=\frac{5}{2}$}

The Pfaffian state is spin polarized and has a chiral edge state. The Lagrangian density for the edge is

$$
L=\frac{2}{4 \pi}\left[\partial_{t} \phi_{1} \partial_{x} \phi_{1}-v_{1}\left(\partial_{x} \phi_{1}\right)^{2}\right]+i \lambda\left(\partial_{t}-v_{\lambda} \partial_{x}\right) \lambda,
$$

where $\phi_{1}$ is a bosonic charged mode and $\lambda$ is a Majorana fermion. We assume the edge state is left moving. The most relevant electron operator is $\psi \propto \lambda e^{-i 2 \phi_{1}}$. The spin-density operators are $S^{x}=S^{y}=0$, and

$$
S^{z}=\frac{1}{2} \psi^{\dagger} \psi \propto \partial_{x} \phi_{1},
$$

where we have used $\lambda^{2}=1$. We find $\mathcal{G}^{x}=\mathcal{G}^{y}=0$, and

$$
\mathcal{G}^{z}(x, t) \propto \frac{1}{\left(x+v_{1} t-i \delta\right)^{2}} .
$$

\section{g. Anti-Pfaffian state at $v=\frac{5}{2}$}

The anti-Pfaffian state is the particle-hole dual of the Pfaffian state. The state is spin polarized. We consider the situation of a clean sample where disorder effect can be neglected and there is translation invariance on the edge. The edge Lagrangian density then takes the form

$$
\begin{aligned}
L=\frac{1}{4 \pi} & {\left[2 \partial_{t} \phi_{\rho} \partial_{x} \phi_{\rho}-\partial_{t} \phi_{n} \partial_{x} \phi_{n}-2 v_{\rho}\left(\partial_{x} \phi_{\rho}\right)^{2}\right.} \\
& \left.\quad-v_{n}\left(\partial_{x} \phi_{n}\right)^{2}-2 v_{\rho n} \partial_{x} \phi_{\rho} \partial_{x} \phi_{n}\right]+i \lambda\left(\partial_{t}+v_{\lambda} \partial_{x}\right) \lambda,
\end{aligned}
$$

where $\phi_{\rho}$ is a left-moving charged boson, $\phi_{n}$ is a right-moving neutral boson, and $\lambda$ is a right-moving Majorana fermion. The edge state is nonchiral. Assuming charge-neutral separation in the edge state, i.e., $v_{\rho} \gg v_{n} \sim v_{\rho n} \sim v_{\lambda}$, we find three most relevant electron operators: $\psi_{1} \propto \lambda e^{-i 2 \phi_{\rho}}, \psi_{2} \propto e^{-i \phi_{n}} e^{-i 2 \phi_{\rho}}$, and $\psi_{3} \propto e^{i \phi_{n}} e^{-i 2 \phi_{\rho}}$. The spin-density operators are $S^{x}=$ $S^{y}=0$ and $S^{z}=S_{f}^{z}+S_{b 1}^{z}+S_{b 2}^{z}$, where

$$
\begin{aligned}
S_{f}^{z} & =\frac{1}{2}\left(\psi_{1}^{\dagger} \psi_{1}+\psi_{2}^{\dagger} \psi_{2}+\psi_{3}^{\dagger} \psi_{3}\right) \propto \partial_{x} \phi_{\rho}, \\
S_{b 1}^{z} & =\frac{1}{2}\left(\psi_{1}^{\dagger} \psi_{2}+\psi_{1}^{\dagger} \psi_{3}+\text { H.c. }\right) \propto e^{i \Delta k x} \lambda e^{i \phi_{n}}+\text { H.c. }, \\
S_{b 2}^{z} & =\frac{1}{2}\left(\psi_{2}^{\dagger} \psi_{3}+\text { H.c. }\right) \propto e^{i \Delta k^{\prime} x} e^{i 2 \phi_{n}}+\text { H.c. },
\end{aligned}
$$

with $\Delta k, \Delta k^{\prime}$ the momentum differences between the edge modes. Upon diagonalizing the edge theory in Eq. (B40), we find $\mathcal{G}^{x}=\mathcal{G}^{y}=0$ and $\mathcal{G}^{z}=\mathcal{G}_{f}^{z}+\mathcal{G}_{b 1}^{z}+\mathcal{G}_{b 2}^{z}$, 
where

$$
\begin{aligned}
& \mathcal{G}_{f}^{z}(x, t) \propto \frac{\tilde{c}_{-}^{2}}{\left(x+v_{+} t-i \delta\right)^{2}}+\frac{\tilde{c}_{+}^{2}}{\left(x-v_{-} t+i \delta\right)^{2}}, \\
& \mathcal{G}_{b 1}^{z}(x, t) \propto \cos (\Delta k x)\left[\frac{1}{x+v_{+} t-i \delta}\right]^{\frac{1}{2} \tilde{c}_{+}^{2}}\left[\frac{1}{x-v_{-} t+i \delta}\right]^{\frac{1}{2} \tilde{c}_{-}^{2}} \\
& \qquad \frac{1}{x-v_{\lambda} t+i \delta}, \\
& \mathcal{G}_{b 2}^{z}(x, t) \propto \cos \left(\Delta k^{\prime} x\right)\left[\frac{1}{x+v_{+} t-i \delta}\right]^{2 \tilde{c}_{+}^{2}}\left[\frac{1}{x-v_{-} t+i \delta}\right]^{2 \tilde{c}_{-}^{2}},
\end{aligned}
$$

where $\tilde{c}_{+}(\theta)=\sqrt{2} \sinh \theta$ and $\tilde{c}_{-}(\theta)=\sqrt{2} \cosh \theta$. The parameters $v_{+} \simeq v_{\rho}, v_{-} \simeq v_{n}$, and $v_{+} \gg v_{-}$. Notice that $\mathcal{G}_{f}^{z}, \mathcal{G}_{b 1}^{z}$ dominate over $\mathcal{G}_{b 2}^{z}$ at long distances.

\section{h. $S U(2)_{2}$ state at $v=\frac{5}{2}$} is

This is a spin-polarized state. The edge Lagrangian density

$$
\begin{gathered}
L=\frac{1}{4 \pi}\left[2 \partial_{t} \phi_{\rho} \partial_{x} \phi_{\rho}+\partial_{t} \phi_{n} \partial_{x} \phi_{n}-2 v_{\rho}\left(\partial_{x} \phi_{\rho}\right)^{2}\right. \\
\left.-v_{n}\left(\partial_{x} \phi_{n}\right)^{2}\right]+i \lambda\left(\partial_{t}-v_{\lambda} \partial_{x}\right) \lambda,
\end{gathered}
$$

where $\phi_{\rho}$ is a charged boson, $\phi_{n}$ is a neutral boson, and $\lambda$ is a Majorana fermion. The edge state is chiral. The most relevant electron operators and the spin-density operators have the same forms as those in the anti-Pfaffian state. However, note that the fields $\phi_{\rho}, \phi_{n}$ here have different origins from those in Eq. (B40). The correlators are found to be $\mathcal{G}^{x}=\mathcal{G}^{y}=0$ and $\mathcal{G}^{z}=\mathcal{G}_{f}^{z}+\mathcal{G}_{b 1}^{z}+\mathcal{G}_{b 2}^{z}$, where

$$
\begin{aligned}
& \mathcal{G}_{f}^{z}(x, t) \propto \frac{1}{\left(x+v_{\rho} t-i \delta\right)^{2}} \\
& \mathcal{G}_{b 1}^{z}(x, t) \propto \cos (\Delta k x) \frac{1}{\left(x+v_{n} t-i \delta\right)\left(x+v_{\lambda} t-i \delta\right)} \\
& \mathcal{G}_{b 2}^{z}(x, t) \propto \cos \left(\Delta k^{\prime} x\right) \frac{1}{\left(x+v_{n} t-i \delta\right)^{4}}
\end{aligned}
$$

with $\Delta k, \Delta k^{\prime}$ the momentum differences between the edge modes.

\section{Time integral}

The QH states we have discussed can be divided into three types.

Type (i): The edge state is chiral and contains one bosonic mode. Examples include Laughlin states at $v=1 / m$ and the Pfaffian state at $v=\frac{5}{2}$. The in-plane correlators vanish, while the out-of-plane correlator has the form

$$
\mathcal{G}^{(\mathrm{i})}(x, t)=\left[\frac{1}{\delta+i(t+x / v)}\right]^{n},
$$

neglecting the proportionality constant and assuming the edge state is left moving, where $n \geqslant 2$ is an even integer and $v>0$ is the speed of the edge mode.

Type (ii): The edge state is chiral and contains multiple interacting bosonic modes. Examples include the QH state at $v=2$ and the 331 state at $v=\frac{5}{2}$. The correlators can have the form of Eq. (B45), or

$$
\mathcal{G}^{(\mathrm{ii})}(x, t)=\left[\frac{1}{\delta+i\left(t+x / v_{+}\right)}\right]^{g_{+}}\left[\frac{1}{\delta+i\left(t+x / v_{-}\right)}\right]^{g_{-}},
$$

neglecting the proportionality constant and the modulating factor, and assuming the edge state is left moving, where $g_{+}$ and $g_{-}$are nonintegers but $g_{+}+g_{-}$is an even integer. From previous calculations, we have $0<g_{+} \ll 1$ and $g_{-}>1$. To a good approximation, $v_{+}$and $v_{-}$can be considered as the speeds of the physical charged mode and neutral mode in the edge state, respectively, so that $v_{+} \gg v_{-}>0$. We have suppressed the spin-component index for simplicity.

Type (iii): The edge state is nonchiral. Examples include the $\mathrm{QH}$ state at $v=\frac{2}{3}$ and the 113 state at $v=\frac{5}{2}$. The correlators can have the form of Eq. (B45), or

$$
\mathcal{G}^{(\mathrm{iii})}(x, t)=\left[\frac{1}{\delta+i\left(t+x / v_{+}\right)}\right]^{g_{+}}\left[\frac{1}{\delta+i\left(t-x / v_{-}\right)}\right]^{g_{-}},
$$

neglecting the proportionality constant and the modulating factor, where $g_{+}, g_{-}$, and $g_{+}+g_{-}$are all nonintegers. We have $0<g_{+} \ll 1$ and $g_{-}>1$. The parameters $v_{+}$and $v_{-}$can again be considered as the speeds of the physical charged mode and neutral mode, respectively, so that $v_{+} \gg v_{-}>0$.

In writing Eqs. (B45)-(B47), we have assumed that there are only two distinct velocities in the system: the chargedmode velocity and the neutral-mode velocity. In particular, for the anti-Pfaffian state we make the approximation that the Majorana fermion and the neutral boson propagate at the same speed. For the $\mathrm{SU}(2)_{2}$ state, there is no need for such an approximation and the correlators take the forms of either Eq. (B45) or (B46).

In the following, we evaluate $I=\int_{0}^{\infty} d t e^{-\eta t} \operatorname{Im} \mathcal{G}^{a}(x, t)$, where $\eta=0^{+}$and $a=$ (i), (ii), (iii).

\section{a. Type (i)}

For type (i) edge states,

$$
\begin{aligned}
I & =\frac{1}{2 i}\left\{\int_{0}^{\infty} d t e^{-\eta t}\left[\frac{1}{\delta+i(t+x / v)}\right]^{n}-\text { c.c. }\right\} \\
& \equiv I_{1}-I_{2} .
\end{aligned}
$$

The integrand of $I_{1}$ has an $n$ th-order pole at $t_{1}=-x / v+i \delta$, while the integrand of $I_{2}$ has an $n$ th-order pole at $t_{2}=-x / v-$ $i \delta$. By residue theorem,

$$
\int_{0}^{\infty} d t \frac{1}{\left(t-t_{k}\right)^{n}}=-\operatorname{Res}\left(\frac{\ln t}{\left(t-t_{k}\right)^{n}} ; t_{k}\right)
$$

where $k=1,2$. This gives

$$
I_{1}=I_{2}=\frac{1}{2 i} \frac{(-1)^{n / 2}}{n-1}\left(\frac{x}{v}\right)^{1-n},
$$

so that $I=0$. This suggests that the spin susceptibility in type (i) edge states vanishes to the lowest order (i.e., considering only the most relevant operators). 


\section{b. Type (ii)}

For type (ii) edge states,

$$
\begin{aligned}
I= & \int_{0}^{\infty} d t e^{-\eta t} \operatorname{Im}\left[\frac{1}{\delta+i\left(t+x / v_{+}\right)}\right]^{g_{+}} \\
& \times\left[\frac{1}{\delta+i\left(t+x / v_{-}\right)}\right]^{g_{-}} .
\end{aligned}
$$

The integrand has two branch points $-x / v_{+}+i \delta$ and $-x / v_{-}+i \delta$. Choosing the branch cut appropriately,

$$
\begin{aligned}
& \operatorname{Im}\left[\frac{1}{\delta+i\left(t+x / v_{+}\right)}\right]^{g_{+}}\left[\frac{1}{\delta+i\left(t+x / v_{-}\right)}\right]^{g_{-}} \\
& =\operatorname{Im}\left\{e^{-i \frac{\pi}{2} g_{+} \operatorname{sgn}\left(t+\frac{x}{v_{+}}\right)} e^{-i \frac{\pi}{2} g_{-} \operatorname{sgn}\left(t+\frac{x}{v_{-}}\right)}\right\}\left|\mathcal{G}^{(\mathrm{ii})}(x, t)\right| \\
& =\Theta\left(t+\frac{x}{v_{+}}\right) \Theta\left(-t-\frac{x}{v_{-}}\right) \sin \left[\frac{\pi}{2}\left(g_{-}-g_{+}\right)\right]\left|\mathcal{G}^{(\mathrm{ii})}(x, t)\right|,
\end{aligned}
$$

where $\Theta(x)$ is the Heaviside step function, $\operatorname{sgn}(x)$ is the signum function, and

$$
\left|\mathcal{G}^{(\mathrm{ii})}(x, t)\right|=\left|t+\frac{x}{v_{+}}\right|^{-g_{+}}\left|t+\frac{x}{v_{-}}\right|^{-g_{-}} .
$$

We have used the fact that $g_{+}+g_{-}$is an even integer, so that $\operatorname{Im}\left\{e^{-i \frac{\pi}{2}\left(g_{+}+g_{-}\right)}\right\}=0$. Notice also that $I=0$ if we set $v_{+}=v_{-}$, which is consistent with the previous result for type (i) edge states. In our scenario, $v_{+} \gg v_{-}>0$. The integral is nonzero only when $x<0$. Explicitly,

$$
\begin{aligned}
I= & \Theta(-x) \sin \left[\frac{\pi}{2}\left(g_{-}-g_{+}\right)\right] \int_{-x / v_{+}}^{-x / v_{-}} d t e^{-\eta t}\left|\mathcal{G}^{(\mathrm{ii})}(x, t)\right| \\
= & \Theta(-x) \sin \left[\frac{\pi}{2}\left(g_{-}-g_{+}\right)\right]\left(\frac{1}{v_{+}}-\frac{1}{v_{-}}\right)^{-g} \\
& \times B\left(1-g_{+}, 1-g_{-}\right)|x|^{-g}
\end{aligned}
$$

where $B(x, y)$ is the Euler beta function and we define $g=$ $g_{+}+g_{-}-1$.

The above calculation applies to left-moving edge states. For right-moving edge states, one replaces $\Theta(-x)$ with $\Theta(x)$, and sends $v_{+}, v_{-} \rightarrow-v_{+},-v_{-}$in Eq. (B54). The exponent $g$ determines the scaling of the spin susceptibility with distance, and may take different values $g^{\alpha}$ for different spin components $\alpha=x, y, z$. For type (ii) edge states, $g^{\alpha}$ are integral invariants depending on the topological order of the bulk QH liquid. For instance, $g^{x}=g^{y}=1$ for the $\mathrm{QH}$ state at $v=2$.

\section{c. Type (iii)}

For type (iii) edge states,

$$
\begin{aligned}
I= & \int_{0}^{\infty} d t e^{-\eta t} \operatorname{Im}\left[\frac{1}{\delta+i\left(t+x / v_{+}\right)}\right]^{g_{+}} \\
& \times\left[\frac{1}{\delta+i\left(t-x / v_{-}\right)}\right]^{g_{-}} .
\end{aligned}
$$

We have

$$
\begin{aligned}
\operatorname{Im} & {\left[\frac{1}{\delta+i\left(t+x / v_{+}\right)}\right]^{g_{+}}\left[\frac{1}{\delta+i\left(t-x / v_{-}\right)}\right]^{g_{-}} } \\
= & \operatorname{Im}\left\{e^{-i \frac{\pi}{2} g_{+} \operatorname{sgn}\left(t+\frac{x}{v_{+}}\right)} e^{-i \frac{\pi}{2} g_{-} \operatorname{sgn}\left(t-\frac{x}{v_{-}}\right)}\right\}\left|\mathcal{G}^{(\mathrm{iii})}(x, t)\right| \\
= & \left\{\left[\Theta\left(-t-\frac{x}{v_{+}}\right)-\Theta\left(-t+\frac{x}{v_{-}}\right)\right] \sin \left[\frac{\pi}{2}\left(g_{+}-g_{-}\right)\right]\right. \\
& \left.-\Theta\left(t+\frac{x}{v_{+}}\right) \Theta\left(t-\frac{x}{v_{-}}\right) \sin \left[\frac{\pi}{2}(g+1)\right]\right\}\left|\mathcal{G}^{(\mathrm{iii})}(x, t)\right|,
\end{aligned}
$$

where

$$
\left|\mathcal{G}^{(\mathrm{iii})}(x, t)\right|=\left|t+\frac{x}{v_{+}}\right|^{-g_{+}}\left|t-\frac{x}{v_{-}}\right|^{-g_{-}} .
$$

The integral is nonzero for both $x>0$ and $x<0$. We find $I=\Theta(x) I_{>}+\Theta(-x) I_{<}$, where

$$
\begin{aligned}
I_{>}= & \sin \left[\frac{\pi}{2}\left(g_{-}-g_{+}\right)\right] \int_{0}^{x / v_{-}} d t e^{-\eta t}\left|\mathcal{G}^{(\mathrm{iii})}(x, t)\right| \\
& -\sin \left[\frac{\pi}{2}(g+1)\right] \int_{x / v_{-}}^{\infty} d t e^{-\eta t}\left|\mathcal{G}^{(\mathrm{iii})}(x, t)\right| \\
= & \left\{\sin \left[\frac{\pi}{2}\left(g_{-}-g_{+}\right)\right] \frac{v_{+}^{g_{+}} v_{-}^{g_{-}-1}}{1-g_{-}} F\left(1, g_{+} ; 2-g_{-} ;-\frac{v_{+}}{v_{-}}\right)\right. \\
& \left.-\sin \left[\frac{\pi}{2}(g+1)\right]\left(\frac{1}{v_{+}}+\frac{1}{v_{-}}\right)^{-g} B\left(g, 1-g_{-}\right)\right\}|x|^{-g},
\end{aligned}
$$

and

$$
\begin{aligned}
I_{<}= & \sin \left[\frac{\pi}{2}\left(g_{+}-g_{-}\right)\right] \int_{0}^{-x / v_{+}} d t e^{-\eta t}\left|\mathcal{G}^{(\mathrm{iii})}(x, t)\right| \\
& -\sin \left[\frac{\pi}{2}(g+1)\right] \int_{-x / v_{+}}^{\infty} d t e^{-\eta t}\left|\mathcal{G}^{(\mathrm{iii})}(x, t)\right| \\
= & \left\{\sin \left[\frac{\pi}{2}\left(g_{+}-g_{-}\right)\right] \frac{v_{+}^{g_{+}-1} v_{-}^{g_{-}}}{1-g_{+}} F\left(1, g_{-} ; 2-g_{+} ;-\frac{v_{-}}{v_{+}}\right)\right. \\
& \left.-\sin \left[\frac{\pi}{2}(g+1)\right]\left(\frac{1}{v_{+}}+\frac{1}{v_{-}}\right)^{-g} B\left(g, 1-g_{+}\right)\right\}|x|^{-g},
\end{aligned}
$$

where $F(a, b ; c ; x)$ is the hypergeometric function. Notice that $I_{>}$and $I_{<}$are related by the exchange of parameters

$$
g_{+} \leftrightarrow g_{-} \quad \text { and } \quad v_{+} \leftrightarrow v_{-},
$$

which technically reverts the chirality of all the edge modes, as seen from Eq. (B47). For type (iii) edge states, $g$ (i.e., $g^{\alpha}$, where $\alpha=x, y, z)$ takes noninteger values. Let us write $g^{\alpha}=$ $g_{0}^{\alpha}+\delta g^{\alpha}$, where $g_{0}^{\alpha}$ is the integer part of $g^{\alpha}$. We find $\delta g^{\alpha} \ll g_{0}^{\alpha}$ for all the type (iii) edge states being discussed. For instance, $\delta g^{x}=\delta g^{y}=4 \sinh ^{2} \theta$, where $\theta \ll 1$, while $g_{0}^{x}=g_{0}^{y}=1$ in the spin-unpolarized QH state at $v=\frac{2}{3}$. 


\section{Full expression of spin susceptibility}

Substituting the above results in Eq. (B2), we obtain the spin susceptibility in $\mathrm{QH}$ edge states

$$
\chi^{\alpha \alpha}(x)=\frac{\cos (\Delta k x)}{4 \pi^{2}} l^{2} a^{g^{\alpha}-1} v_{+}^{-g_{+}^{\alpha}} v_{-}^{-g_{-}^{\alpha}} \times I,
$$

where we have restored the spin-component index and the proportionality constant. The short-distance cutoff $a$ can be taken as the lattice constant of the host material of the QH system. For left-moving type (ii) edge states, I is given by Eq. (B54). We have $\chi^{\alpha \alpha}(x)=\cos (\Delta k x)|x|^{-g^{\alpha}} \Theta(-x) C^{\alpha}\left(g^{\alpha}, \boldsymbol{v}\right)$, where $\boldsymbol{g}^{\alpha}=$ $\left(g_{+}^{\alpha}, g_{-}^{\alpha}\right), \boldsymbol{v}=\left(v_{+}, v_{-}\right)$, and

$$
\begin{aligned}
C^{\alpha}\left(\boldsymbol{g}^{\alpha}, \boldsymbol{v}\right)= & \frac{l^{2} a^{g^{\alpha}-1}}{4 \pi^{2}} \sin \left[\frac{\pi}{2}\left(g_{-}^{\alpha}-g_{+}^{\alpha}\right)\right] \frac{v_{+}^{g_{-}^{\alpha}-1} v_{-}^{g_{+}^{\alpha}-1}}{\left(v_{-}-v_{+}\right)^{g^{\alpha}}} \\
& \times B\left(1-g_{+}^{\alpha}, 1-g_{-}^{\alpha}\right) .
\end{aligned}
$$

For type (iii) edge states, $I$ is given by Eqs. (B58) and (B59). We have $\chi^{\alpha \alpha}(x)=\cos (\Delta k x)|x|^{-g^{\alpha}}\left\{\Theta(x) C_{>}^{\alpha}\left(g^{\alpha}, \boldsymbol{v}\right)+\right.$ $\left.\Theta(-x) C_{<}^{\alpha}\left(g^{\alpha}, v\right)\right\}$, where

$$
\begin{aligned}
& C_{>}^{\alpha}\left(\boldsymbol{g}^{\alpha}, \boldsymbol{v}\right)=\frac{l^{2} a^{g^{\alpha}-1}}{4 \pi^{2}}\left\{\sin \left[\frac{\pi}{2}\left(g_{-}^{\alpha}-g_{+}^{\alpha}\right)\right] \frac{v_{-}^{-1}}{1-g_{-}^{\alpha}} F\left(1, g_{+}^{\alpha} ; 2-g_{-}^{\alpha} ;-\frac{v_{+}}{v_{-}}\right)-\sin \left[\frac{\pi}{2}\left(g^{\alpha}+1\right)\right] \frac{v_{+}^{g_{-}^{\alpha}-1} v_{-}^{g_{+}^{\alpha}-1}}{\left(v_{+}+v_{-}\right)^{g^{\alpha}}} B\left(g^{\alpha}, 1-g_{-}^{\alpha}\right)\right\} \\
& C_{<}^{\alpha}\left(\boldsymbol{g}^{\alpha}, \boldsymbol{v}\right)=\frac{l^{2} a^{g^{\alpha}-1}}{4 \pi^{2}}\left\{\sin \left[\frac{\pi}{2}\left(g_{+}^{\alpha}-g_{-}^{\alpha}\right)\right] \frac{v_{+}^{-1}}{1-g_{+}^{\alpha}} F\left(1, g_{-}^{\alpha} ; 2-g_{+}^{\alpha} ;-\frac{v_{-}}{v_{+}}\right)-\sin \left[\frac{\pi}{2}\left(g^{\alpha}+1\right)\right] \frac{v_{+}^{g_{-}^{\alpha}-1} v_{-}^{g_{+}^{\alpha}-1}}{\left(v_{+}+v_{-}\right)^{g^{\alpha}}} B\left(g^{\alpha}, 1-g_{+}^{\alpha}\right)\right\} .
\end{aligned}
$$

We see that $C_{>}^{\alpha}\left(\boldsymbol{g}^{\alpha}, \boldsymbol{v}\right)$ and $C_{<}^{\alpha}\left(\boldsymbol{g}^{\alpha}, \boldsymbol{v}\right)$ are related by the exchange of arguments: $g_{+}^{\alpha} \leftrightarrow g_{-}^{\alpha}$ and $v_{+} \leftrightarrow v_{-}$.

Equations (B62) and (B63) show that the RKKY interaction is ferromagnetic at short distances. To estimate the strength of the RKKY interaction, we extract the dimensional part $\left[\chi^{\alpha \alpha}(x)\right]$ of the spin susceptibility. For type (ii) edge states,

$$
\left[\chi^{\alpha \alpha}(x)\right]=l^{2} a^{g^{\alpha}-1} \frac{v_{+}^{g_{-}^{\alpha}-1} v_{-}^{g_{+}^{\alpha}-1}}{\left(v_{+}-v_{-}\right)^{g^{\alpha}}}|x|^{-g^{\alpha}} .
$$

For type (iii) edge states, there are multiple terms in $\chi^{\alpha \alpha}(x)$, with

$$
\begin{aligned}
{\left[\chi^{\alpha \alpha}(x)\right]=} & l^{2} a^{g^{\alpha}-1} \frac{v_{+}^{g_{-}^{\alpha}-1} v_{-}^{g_{+}^{\alpha}-1}}{\left(v_{+}+v_{-}\right)^{g^{\alpha}}}|x|^{-g^{\alpha}} ; \\
& l^{2} a^{g^{\alpha}-1} v_{-}^{-1}|x|^{-g^{\alpha}} ; l^{2} a^{g^{\alpha}-1} v_{+}^{-1}|x|^{-g^{\alpha}} .
\end{aligned}
$$

Using $0<g_{+}^{\alpha} \ll 1$ and $v_{+} \gg v_{-}$, we find

$$
\left[\chi^{\alpha \alpha}(x)\right] \simeq l^{2} a^{g^{\alpha}-1} v_{-}^{-1}|x|^{-g^{\alpha}}
$$

for both type (ii) and type (iii) edge states.

\section{APPENDIX C: EXCHANGE}

Here, we estimate the strength of the exchange interaction between the QD electron and the electrons in the edge modes. The textbook formula gives the exchange integral as

$$
J=C \int d \mathbf{r}_{1} d \mathbf{r}_{2} \Psi_{1}^{*}\left(\mathbf{r}_{1}\right) \Psi_{2}^{*}\left(\mathbf{r}_{2}\right) \frac{1}{\left|\mathbf{r}_{1}-\mathbf{r}_{2}\right|} \Psi_{1}\left(\mathbf{r}_{2}\right) \Psi_{2}\left(\mathbf{r}_{1}\right)
$$

for two particles in single-particle orbitals $\Psi_{1}, \Psi_{2}$, interacting through an unscreened Coulomb interaction parametrized by $C=\frac{e^{2}}{4 \pi \epsilon_{0} \epsilon_{r}}$, where $e$ is the elementary charge, $\epsilon_{0}$ the vacuum permittivity, and $\epsilon_{r}$ the relative permittivity of the medium.
Providing a microscopic theory of the exchange for our case is well beyond the scope of this paper. Instead, we are interested only in the interaction strength scale. To get a rough estimate, let us assume that the exchange interaction is local

$$
J=\beta \int d \mathbf{r}_{1} d \mathbf{r}_{2} \Psi_{1}^{*}\left(\mathbf{r}_{1}\right) \Psi_{2}^{*}\left(\mathbf{r}_{2}\right) \delta\left(\mathbf{r}_{1}-\mathbf{r}_{2}\right) \Psi_{1}\left(\mathbf{r}_{2}\right) \Psi_{2}\left(\mathbf{r}_{1}\right),
$$

which transforms the equation into a density-density interaction

$$
J=\beta \int d \mathbf{r} \rho_{1}(\mathbf{r}) \rho_{2}(\mathbf{r}) .
$$

One can explicitly evaluate Eq. (C1) for a tunnel-coupled double dot modeled by a 2D harmonic confinement [54] and then compare to the result given by Eq. (C3). The calculated energies scale the same with the interdot distance, and the overall prefactors are related by $\beta=C l$, with $l$ the confinement length of the dot potential. We further guide ourselves by experiments, which measured the exchange energy in fewelectron QDs made in 2DEG in GaAs. The maximal scale $C / l$, which evaluates to $\simeq 3 \mathrm{meV}$ for typical GaAs parameters $\epsilon_{r}=12.9$ and $l=30 \mathrm{~nm}$, is indeed approached in a single dot where the densities overlap in Eq. (C3) is of order 1 in dimensionless units $\left(l^{-2}\right)$. A suppression of the interdot tunneling (by increasing the interdot distance) leads to a decreasing exchange, which reaches $J_{D D} \simeq 0.1-0.01 \mathrm{meV}$ in a tunnel-coupled double dot. Assuming that an analogous suppression will result from the tunnel coupling of our dot coupled to the edge finally gives $J_{D D}$ as an order of magnitude estimate for $\Gamma$, which we used in the main text as the coupling constant between an electron spin in a QD and a quasi-1D spin density of the edge.
[1] M. A. Nielsen and I. L. Chuang, Quantum Computation and Quantum Information (Cambridge University Press, Cambridge, 2000).
[2] D. Loss and D. DiVincenzo, Quantum computation with quantum dots, Phys. Rev. A 57, 120 (1998). 
[3] C. Kloeffel and D. Loss, Prospects for spin-based quantum computing in quantum dots, Annu. Rev. Condens. Matter Phys. 4, 51 (2013).

[4] H. Bluhm, S. Foletti, I. Neder, M. Rudner, D. Mahalu, V. Umansky, and A. Yacoby, Dephasing time of GaAs electron-spin qubits coupled to a nuclear bath exceeding $200 \mu$ s, Nat. Phys. 7 , 109 (2011).

[5] K. M. Svore, B. M. Terhal, and D. P. DiVincenzo, Local fault-tolerant quantum computation, Phys. Rev. A 72, 022317 (2005).

[6] A. M. Childs, H. L. Haselgrove, and M. A. Nielsen, Lower bounds on the complexity of simulating quantum gates, Phys. Rev. A 68, 052311 (2003).

[7] A. Imamog-lu, D. D. Awschalom, G. Burkard, D. P. DiVincenzo, D. Loss, M. Sherwin, and A. Small, Quantum Information Processing Using Quantum Dot Spins and Cavity QED, Phys. Rev. Lett. 83, 4204 (1999).

[8] L. Childress, A. S. Sørensen, and M. D. Lukin, Mesoscopic cavity quantum electrodynamics with quantum dots, Phys. Rev. A 69, 042302 (2004).

[9] G. Burkard and A. Imamoglu, Ultra-long-distance interaction between spin qubits, Phys. Rev. B 74, 041307(R) (2006).

[10] M. Trif, V. N. Golovach, and D. Loss, Spin dynamics in InAs nanowire quantum dots coupled to a transmission line, Phys. Rev. B 77, 045434 (2008).

[11] L. Trifunovic, O. Dial, M. Trif, J. R. Wootton, R. Abebe, A. Yacoby, and D. Loss, Long-distance spin-spin coupling via floating gates, Phys. Rev. X 2, 011006 (2012).

[12] L. Trifunovic, F. L. Pedrocchi, and D. Loss, Long-Distance Entanglement of Spin Qubits via Ferromagnet, Phys. Rev. X 3, 041023 (2013).

[13] P. Stano, J. Klinovaja, F. R. Braakman, L. M. K. Vandersypen, and D. Loss, Fast long-distance control of spin qubits by photon-assisted cotunneling, Phys. Rev. B 92, 075302 (2015).

[14] M. A. Ruderman and C. Kittel, Indirect exchange coupling of nuclear magnetic moments by conduction electrons, Phys. Rev. 96, 99 (1954).

[15] T. Kasuya, A theory of metallic ferro- and antiferromagnetism on zener's model, Prog. Theor. Phys. 16, 45 (1956).

[16] K. Yosida, Magnetic properties of Cu-Mn alloys, Phys. Rev. 106, 893 (1957).

[17] X.-G. Wen, Quantum Field Theory of Many-Body Systems: From the Origin of Sound to an Origin of Light and Electrons (Oxford University Press, New York, 2004).

[18] H. Kiyama, T. Nakajima, S. Teraoka, A. Oiwa, and S. Tarucha, Spin-dependent current through a quantum dot from spinpolarized nonequilibrium quantum Hall edge channels, Phys. Rev. B 91, 155302 (2015).

[19] P. Stano, J. Klinovaja, A. Yacoby, and D. Loss, Local spin susceptibilities of low-dimensional electron systems, Phys. Rev. B 88, 045441 (2013).

[20] J. R. Schrieffer and P. A. Wolff, Relation between the anderson and Kondo hamiltonians, Phys. Rev. 149, 491 (1966).

[21] S. Bravyi, D. P. DiVincenzo, and D. Loss, Schrieffer-Wolff Transformation for quantum many-body systems, Ann. Phys. (Amsterdam) 326, 2793 (2011).

[22] T. Giamarchi, Quantum Physics in One Dimension (Oxford University Press, New York, 2003).
[23] B. Braunecker, P. Simon, and D. Loss, Nuclear magnetism and electron order in interacting one-dimensional conductors, Phys. Rev. B 80, 165119 (2009).

[24] Y.-W. Lee and Y.-L. Lee, Electrical control and interaction effects of the RKKY interaction in helical liquids, Phys. Rev. B 91, 214431 (2015).

[25] E. Bocquillon, V. Freulon, J-.M Berroir, P. Degiovanni, B. Plaçais, A. Cavanna, Y. Jin, and G. Fève, Separation of neutral and charge modes in one-dimensional chiral edge channels, Nat. Commun. 4, 1839 (2013).

[26] A. Bid, N. Ofek, H. Inoue, M. Heiblum, C. L. Kane, V. Umansky, and D. Mahalu, Observation of neutral modes in the fractional quantum Hall regime, Nature (London) 466, 585 (2010).

[27] S. M. Girvin, Particle-Hole Symmetry in the anomalous quantum Hall effect, Phys. Rev. B 29, 6012(R) (1984).

[28] C. Wang and D. E. Feldman, Chirality, Causality, and Fluctuation-Dissipation Theorems in Nonequilibrium Steady States, Phys. Rev. Lett. 110, 030602 (2013).

[29] J. P. Eisenstein, H. L. Stormer, L. N. Pfeiffer, and K. W. West, Evidence for a spin transition in the $v=2 / 3$ fractional quantum Hall effect, Phys. Rev. B 41, 7910(R) (1990).

[30] B. I. Halperin, Theory of the quantized Hall conductance, Helv. Phys. Acta. 56, 75 (1983).

[31] G. Yang and D. E. Feldman, Experimental constraints and a possible quantum Hall state at $v=5 / 2$, Phys. Rev. B 90, 161306(R) (2014).

[32] G. Moore and N. Read, Nonabelions in the fractional quantum Hall effect, Nucl. Phys. B 360, 362 (1991).

[33] M. Levin, B. I. Halperin, and B. Rosenow, Particle-Hole Symmetry and the Pfaffian State, Phys. Rev. Lett. 99, 236806 (2007).

[34] S.-S. Lee, S. Ryu, C. Nayak, and M. P. A. Fisher, Particle-Hole Symmetry and the $v=\frac{5}{2}$ Quantum Hall State, Phys. Rev. Lett. 99, 236807 (2007).

[35] X.-G. Wen, Non-Abelian Statistics in the Fractional Quantum Hall States, Phys. Rev. Lett. 66, 802 (1991).

[36] D. B. Chklovskii, B. I. Shklovskii, and L. I. Glazman, Electrostatics of edge channels, Phys. Rev. B 46, 4026 (1992).

[37] J. P. Eisenstein, L. N. Pfeiffer, and K. W. West, Independently contacted two-dimensional electron systems in double quantum wells, Appl. Phys. Lett. 57, 2324 (1990).

[38] O. M. Auslaender, A. Yacoby, R. de Picciotto, K. W. Baldwin, L. N. Pfeiffer, and K. W. West, Experimental Evidence for Resonant Tunneling in a Luttinger Liquid, Phys. Rev. Lett. 84, 1764 (2000).

[39] L. Pfeiffer, H. L. Störmer, K. W. Baldwin, K. W. West, A R. Goñi, A. Pinczuk, R. C. Ashoori, M. M. Dignam, and W. Wegscheider, Cleaved edge overgrowth for quantum wire fabrication, J. Cryst. Growth 127, 849 (1993).

[40] C. P. Scheller, T.-M. Liu, G. Barak, A. Yacoby, L. N. Pfeiffer, K. W. West, and D. M. Zumbühl, Possible Evidence for Helical Nuclear Spin Order in GaAs Quantum Wires, Phys. Rev. Lett. 112, 066801 (2014).

[41] T. Meng, P. Stano, J. Klinovaja, and D. Loss, Helical nuclear spin order in a strip of stripes in the quantum Hall regime, Eur. Phys. J. B 87, 203 (2014).

[42] L. Zhou, J. Wiebe, S. Lounis, E. Vedmedenko, F. Meier, S. Blügel, P. H. Dederichs, and R. Wiesendanger, Strength 
and directionality of surface Ruderman-Kittel-Kasuya-Yosida interaction mapped on the atomic scale, Nat. Phys. 6, 187 (2010).

[43] J. R. Petta, A. C. Johnson, J. M. Taylor, E. A. Laird, A. Yacoby, M. D. Lukin, C. M. Marcus, M. P. Hanson, and A. C. Gossard, Coherent manipulation of coupled electron spins in semiconductor quantum dots, Science 309, 2180 (2005).

[44] R. Hanson, L. P. Kouwenhoven, J. R. Petta, S. Tarucha, and L. M. K. Vandersypen, Spins in few-electron quantum dots, Rev. Mod. Phys. 79, 1217 (2007).

[45] C. Barthel, D. J. Reilly, C. M. Marcus, M. P. Hanson, and A. C. Gossard, Rapid Single-Shot Measurement of a Singlet-Triplet Qubit, Phys. Rev. Lett. 103, 160503 (2009).

[46] X.-G. Wen, Edge transport properties of the fractional quantum Hall states and weak-impurity scattering of a one-dimensional charge-density wave, Phys. Rev. B 44, 5708 (1991).

[47] G. Yang and D. E. Feldman, Influence of device geometry on tunneling in the $v=\frac{5}{2}$ quantum Hall liquid, Phys. Rev. B 88, 085317 (2013).
[48] A. Stern and B. I. Halperin, Proposed Experiments to Probe the Non-Abelian $v=5 / 2$ Quantum Hall State, Phys. Rev. Lett. 96, 016802 (2006).

[49] P. Bonderson, A. Kitaev, and K. Shtengel, Detecting NonAbelian Statistics in the $v=5 / 2$ Fractional Quantum Hall State, Phys. Rev. Lett. 96, 016803 (2006).

[50] D. E. Feldman and A. Kitaev, Detecting Non-Abelian Statistics with an Electronic Mach-Zehnder Interferometer, Phys. Rev. Lett. 97, 186803 (2006).

[51] C. Wang and D. E. Feldman, Identification of 331 quantum Hall states with Mach-Zehnder interferometry, Phys. Rev. B 82, 165314 (2010).

[52] G. Yang, Probing the $v=\frac{5}{2}$ quantum Hall state with electronic Mach-Zehnder interferometry, Phys. Rev. B 91, 115109 (2015).

[53] M. R. Geller and D. Loss, Aharonov-Bohm effect in the Chiral Luttinger liquid, Phys. Rev. B 56, 9692 (1997).

[54] D. Stepanenko, M. Rudner, B. I. Halperin, and D. Loss, Singlet-triplet splitting in double quantum dots due to spinorbit and hyperfine interactions, Phys. Rev. B 85, 075416 (2012). 